\title{
The Parkinson-associated protein PINK1 interacts with Beclin1 and promotes autophagy
}

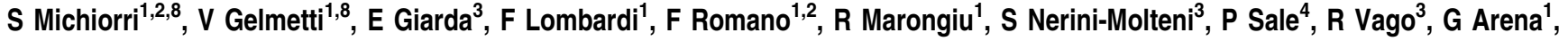 \\ L Torosantucci ${ }^{1}$, L Cassina ${ }^{3}$, MA Russo ${ }^{2}$, B Dallapiccola ${ }^{1}$, EM Valente ${ }^{\star, 1,5}$ and G Casari ${ }^{\star 3,6,7}$
}

Mutations in the PINK1 gene cause autosomal recessive Parkinson's disease. The PINK1 gene encodes a protein kinase that is mitochondrially cleaved to generate two mature isoforms. In addition to its protective role against mitochondrial dysfunction and apoptosis, PINK1 is also known to regulate mitochondrial dynamics acting upstream of the PD-related protein Parkin. Recent data showed that mitochondrial Parkin promotes the autophagic degradation of dysfunctional mitochondria, and that stable PINK1 silencing may have an indirect role in mitophagy activation. Here we report a new interaction between PINK1 and Beclin1, a key pro-autophagic protein already implicated in the pathogenesis of Alzheimer's and Huntington's diseases. Both PINK1 $\mathrm{N}$ - and C-terminal are required for the interaction, suggesting that full-length PINK1, and not its cleaved isoforms, interacts with Beclin1. We also demonstrate that PINK1 significantly enhances basal and starvation-induced autophagy, which is reduced by knocking down Beclin1 expression or by inhibiting the Beclin1 partner Vps34. A mutant, PINK1 ${ }^{\text {W437X }}$, interaction of which with Beclin1 is largely impaired, lacks the ability to enhance autophagy, whereas this is not observed for PINK $1^{\mathrm{G} 309 \mathrm{D}}$, a mutant with defective kinase activity but unaltered ability to bind Beclin1. These findings identify a new function of PINK1 and further strengthen the link between autophagy and proteins implicated in the neurodegenerative process.

Cell Death and Differentiation (2010) 17, 962-974; doi:10.1038/cdd.2009.200; published online 8 January 2010

Parkinson's disease (PD) is a frequent neurodegenerative disorder resulting from massive degeneration of the dopaminergic neurons in the substantia nigra. Although most cases are sporadic, several genes are known to cause familial PD, especially with early onset. ${ }^{1}$

Mutations in the PINK1 gene are the second most frequent cause of autosomal recessive PD after those in the Parkin gene. ${ }^{2,3}$ The PINK1 gene encodes a serine-threonine kinase with an $\mathrm{N}$-terminal mitochondrial import sequence, first characterized as a protein aimed at maintaining mitochondrial integrity and preventing apoptosis in response to cellular stressors. $^{2,4-8}$ This neuroprotective role is partly exerted through phosphorylation of the mitochondrial chaperon, TRAP1, although cytoplasm-restricted PINK1 was also shown to protect against MPTP damage. ${ }^{9,10}$ The full-length PINK1 (PINK1-FL) is processed within mitochondria to generate two mature proteins; ${ }^{4,11}$ all three isoforms localize both to the mitochondria and cytosol, their relative ratio being regulated by several factors. ${ }^{10-13}$

Increasing data have demonstrated that absence of functional PINK1 induces abnormalities of mitochondrial morphology. ${ }^{6,14,15}$ In several studies (mostly in Drosophila), PINK1 was shown to promote fission acting upstream of the Fis1-Drp1 machinery, and the mitochondrial phenotype observed in PINK1 knockout flies or silenced cells was associated to reduced fission. ${ }^{16,17}$ Subsequent studies in mammalian cell systems contradicted these results, demonstrating that mutant or silenced PINK1 resulted in increased fragmentation. ${ }^{15,17-19}$

A major progress in this field has come from the finding that PINK1 interacts with Parkin to regulate mitochondrial dynamics. Parkin overexpression was found to rescue the mitochondrial abnormalities observed in PINK1 knockout flies and silenced cells but not vice versa, suggesting that Parkin may act downstream of PINK1 in a common pathway. ${ }^{14,15,19}$ It has been recently shown that Parkin is directly recruited to dysfunctional mitochondria mediating their elimination through mitophagy, ${ }^{20}$ and that stable silencing of PINK1 induces an increase in oxidative stress levels, which activates both mitophagy and mitochondrial fission. ${ }^{19}$ Mitophagy is a tightly regulated autophagy-based mechanism that has a central role in mitochondria quality control, by selectively

\footnotetext{
${ }^{1}$ Casa Sollievo della Sofferenza Hospital, CSS-Mendel Institute, Rome, Italy; ${ }^{2}$ Department of Experimental Medicine, Sapienza University, Rome, Italy; ${ }^{3}$ Human Molecular Genetics Unit, San Raffaele Scientific Institute, DIBIT, Milan, Italy; ${ }^{4}$ IRCCS San Raffaele Pisana, Rome, Italy; ${ }^{5}$ Department of Medical and Surgical Paediatric Sciences, University of Messina, Messina, Italy; ${ }^{6}$ Vita-Salute San Raffaele University School of Medicine, Milan, Italy and ${ }^{7}$ National Institute of Neurosciences, Milan, Italy

${ }^{*}$ Correspondening authors: EM Valente or G Casari, Neurogenetics Unit, CSS-Mendel Institute, viale Regina Margherita 261, 00198 Rome, Italy.

Tel: + 3906 44160537; Fax: + 3906 44160548; E-mail: e.valente@css-mendel.it or casari.giorgio@ @sr.it

${ }^{8}$ These authors contributed equally to this work.

Keywords: PINK1; Beclin1; Parkinson's disease; autophagy; mitochondria

Abbreviations: 3MA, 3-methyladenine; aa, amino acid; ER, endoplasmic reticulum; FL, full length; FLIM, Fluorescence Lifetime Imaging Microscopy; FRET, Fluorescence Resonance Energy Transfer; LC3, Microtubule-associated protein light chain 3; Mfn1, Mitofusin 1; MPTP, 1-methyl-4-phenyl-1,2,3, 6-tetrahydropyridine; OMM, outer mitochondrial membrane; PD, Parkinson's disease; PI3K, phosphatidylinositol 3'-kinase; PINK1, PTEN induced putative kinase 1; TRAP1, TNF receptorassociated protein 1

Received 02.4.09; revised 06.11.09; accepted 17.11.09; Edited by M Piacentini; published online 08.1.10
} 
eliminating damaged mitochondria before they could activate apoptosis. ${ }^{21}$ Indeed, autophagy is being increasingly recognized as an essential homeostatic process to clear misfolded or aggregated proteins and to ensure organellar turnover. The role of autophagy seems even more relevant in aging non-mitotic cells, such as neurons, in which damaged cellular components cannot be diluted through recurring cell division cycles. ${ }^{22}$

Compelling evidence now indicates a protective role of autophagy against neurodegeneration. Dysfunctional autophagy has been implicated in a growing number of neurodegenerative diseases, including PD, that share the pathogenetic pathways of mitochondrial abnormalities and misfolded protein damage. ${ }^{22-24}$ Interestingly, the fission protein Fis1 was found to activate the autophagy pathway, ${ }^{25}$ and it has been proposed that mitochondria fusion/fission machinery and mitophagy could represent a 'quality control axis', regulating mitochondrial dynamics and function. ${ }^{26}$ Although in PINK1-deficient cells mitophagy seems to depend on mitochondrial fission, ${ }^{19}$ Parkin-activated mitophagy seems independent from its effects on mitochondrial morphology, suggesting that the two mechanisms can be regulated together or in parallel. ${ }^{20}$
Here, we present evidence that PINK1-FL, but not its cleaved isoforms or a mutant protein truncated at the C-terminus, interacts with the pro-autophagic protein Beclin1 and enhances autophagy. This effect results from an increase of the autophagic flux and is, at least, partly mediated through the Beclin1-Vps34 complex, suggesting a main role for PINK1 in this fundamental cellular pathway.

\section{Results}

PINK1 interacts with the pro-autophagic protein Beclin1. In search of PINK1 interactors using a yeast twohybrid approach, we screened a human brain cDNA library and obtained a strong interaction with the PINK1 N-terminal region (aa 1-100; Figure 1a). Sequencing of the prey cDNA and subsequent bioinformatic analysis showed that it corresponded to the BECN1 gene, encoding Beclin1.

To confirm the yeast two-hybrid data, we performed in vitro GST pull down using different PINK1 constructs, and detected a strong interaction between PINK1-FL and Beclin1. The interaction was mildly reduced on removing the first 77 amino acids of PINK1, but less than halved on removing the whole a

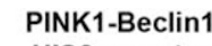
HIS3 reporter

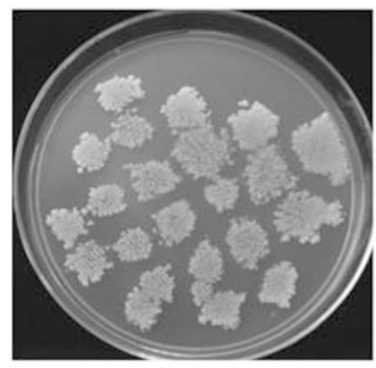

PINK1-Beclin1 ADE2 reporter

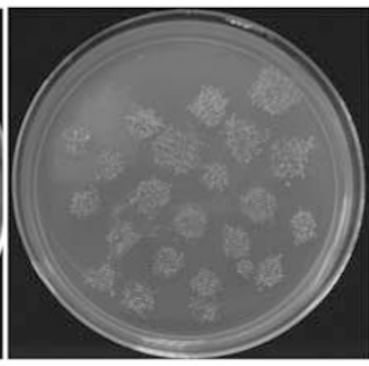

Ponceau staining

b
UN-Beclin1 HIS3 reporter

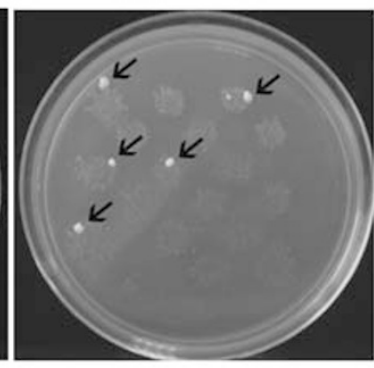

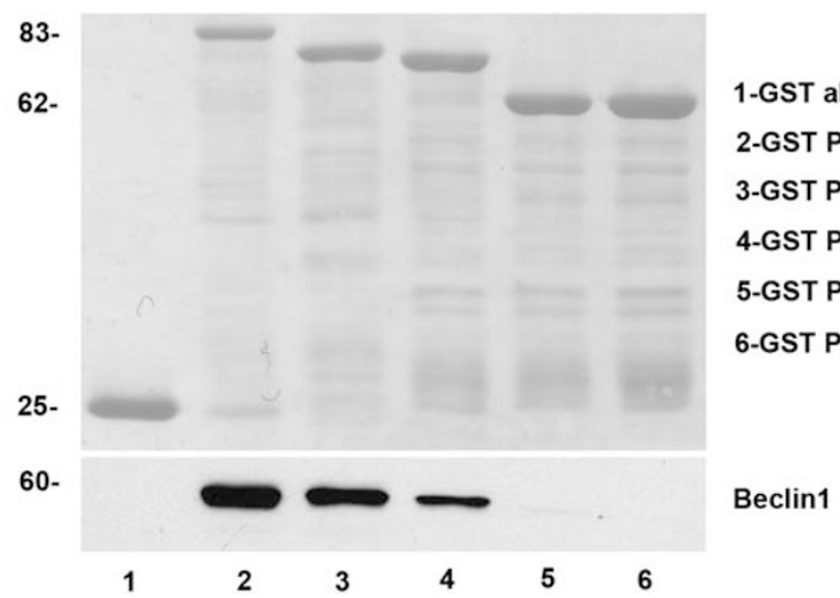

Figure 1 Interaction between PINK1-FL and Beclin1 in different systems. (a) Yeast two-hybrid system showing interaction between the bait encoding for N-terminal region of PINK1 (aa 1-100) and the prey encoding for Beclin1. The prey identified by library screening was cloned in a fresh yeast host, and several independent colonies were array-mated with the original bait strain. After establishment of the diploid strains and replica plating, interaction testing was confirmed by growth on selective medium for the HIS3 and ADE2 reporter genes (left and middle panel). No growth was observed after mating of the prey with an unrelated bait strain (UR, right panel). Growth of single colonies (arrows) was likely dependent on occasional mutations occurred in the bait or prey. (b) Pull-down assay using purified GST alone, GST-PINK1-FL- and GST-PINK1deleted constructs. Ponceau staining shows the amount of fusion protein used in each pull down 
N-terminus (1-112 aa), and completely abolished when the $\mathrm{C}$-terminus was also deleted (Figure 1b). These results confirmed that PINK1-FL binds Beclin1 in vitro, and suggested that the interaction involves both its $\mathrm{N}$ - and $\mathrm{C}$-terminal regions. To assess whether Beclin1 could be a substrate of PINK1, we performed an in vitro kinase assay using GST-PINK1 $1^{77-581}$ and observed no phosphorylation of Beclin1 (data not shown).

Deletion of PINK1 N- or C-terminal regions, but not loss of its kinase activity, impairs Beclin1 binding in vivo. To test the interaction between PINK1-FL and Beclin1 in vivo, we performed co-immunoprecipitation experiments in HeLa cells co-transfected with HA-tagged PINK1 and Beclin1. In line with pull-down results, we showed that PINK1-FL strongly co-immunoprecipitated Beclin1 (Figure 2a). As no reliable antibodies are available to immunoprecipitate endogenous PINK1, 8,27 we next demonstrated that endogenous Beclin1 specifically co-immunoprecipitated PINK1-FL, whereas no bands were observed corresponding to PINK1-cleaved isoforms (Figure 2b). The binding between Beclin1 and PINK1-FL was further confirmed by immunoprecipitating endogenous Beclin1 in SH-SY5Y cells stably expressing PINK1-FL (Figure 2c).

We then generated several HA-tagged PINK1-deleted and mutant constructs. We selected two PINK1 pathogenic mutations (p.W437X and p.G309D), ${ }^{2}$ with similar stability and subcellular localization in comparison with the wild-type protein. ${ }^{28}$ Mutant PINK1 ${ }^{\text {W437X }}$ lacks the C-terminus and part of the kinase domain, yet it partly maintains the ability to phosphorylate specific substrates such as TRAP1. Conversely, kinase activity is lost by the missense mutant $\mathrm{PINK} 1^{\mathrm{G} 309 \mathrm{D}} 9$ PINK1 constructs lacking either the $\mathrm{N}$-terminus (PINK1 ${ }^{112-581}$ ) or the C-terminus (PINK1 ${ }^{1-496}$, mutant PINK1 ${ }^{\text {W437X }}$ ) showed a marked reduction of Beclin1 co-immunoprecipitation. Conversely, PINK1 G309D_Beclin1 co-immunoprecipitation was comparable with PINK1-FL (Figure 2d).

To validate these results in a different experimental approach, we used fluorescence lifetime imaging microscopy (FLIM), a confocal microscopy-based fluorescence resonance energy transfer (FRET) technique, which can reveal protein-protein interactions in vivo. We performed FLIM on HeLa cells transiently co-transfected with PINK1-FL, deleted or mutant constructs and Beclin1. Immunostaining of PINK1$\mathrm{FL}$ with donor fluorophore generated a lifetime value that markedly decreased when Beclin1 also was costained with the acceptor fluorophore, indicating interaction of the two proteins. A similar reduction of donor lifetime could be observed on co-staining PINK $1^{\mathrm{G} 309 \mathrm{D}}$ and Beclin1; conversely, co-immunostaining PINK $1^{112-581}$, PINK $1^{1-496}$ and $\mathrm{PINK} 1^{\mathrm{W} 437 \mathrm{X}}$ with Beclin1 did not result in significant reductions of donor lifetime. To assess FLIM specificity, we performed a further experiment testing PINK1-FL and Calnexin, a marker of the endoplasmic reticulum (ER) known not to co-localize with PINK1, but could observe no interaction (Figure 3). Equivalent results were obtained in all experiments when acceptor and donor were exchanged (data not shown).

Taken together, co-immunoprecipitation and FLIM experiments confirmed that Beclin1 strongly interacts with
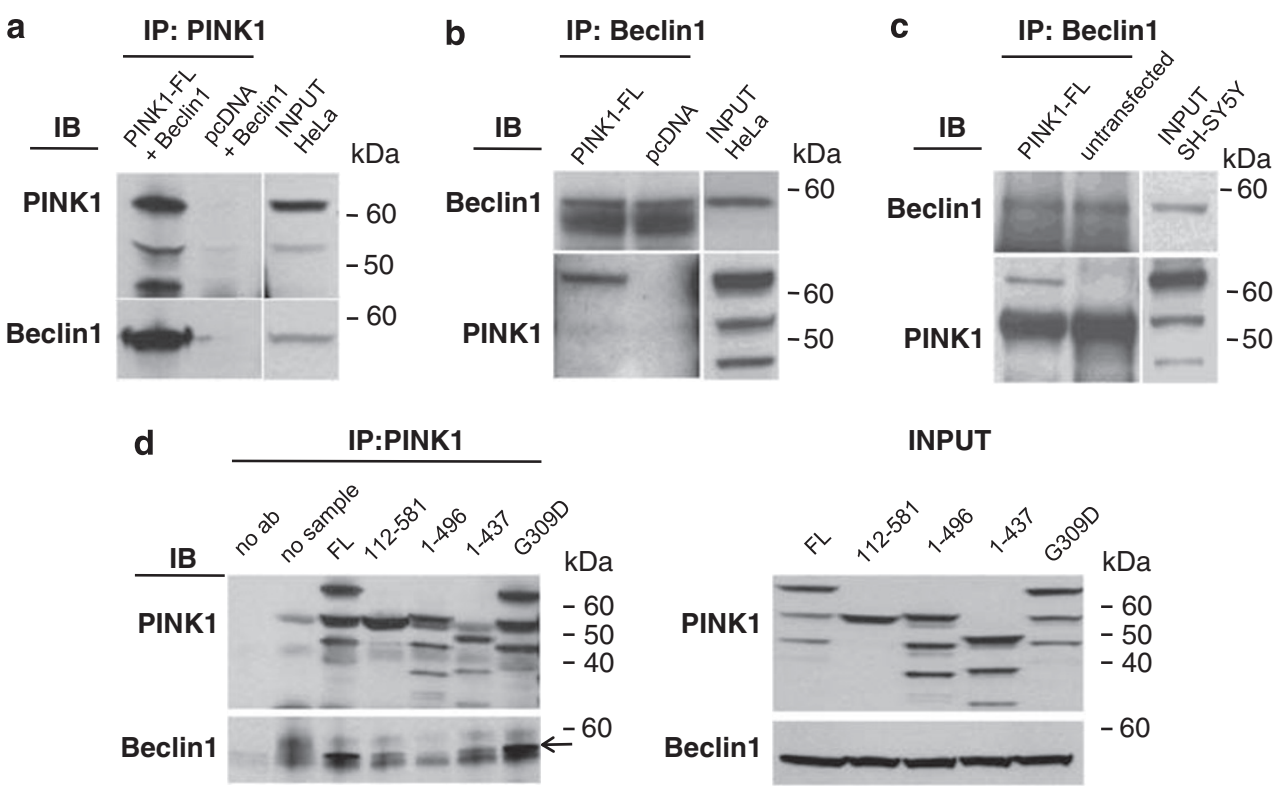

Figure 2 Co-immunoprecipitation (co-IP) between PINK1 and Beclin1. (a) Co-IP of overexpressed Beclin1 and PINK1-FL in HeLa cells. IP was performed with rat anti-HA antibody (PINK1) followed by immunoblotting with rabbit anti-Beclin1 antibody (Beclin1) and rabbit anti-HA antibody. The three bands detected with anti-HA antibody correspond to PINK1-FL- (upper band) and PINK1-cleaved isoforms (middle and lower bands). Control sample is represented by HeLa cells transfected with pcDNA3.1 empty vector and Beclin1. (b) Co-IP of endogenous Beclin1 and overexpressed PINK1-FL in HeLa cells. IP was performed with rabbit anti-Beclin1 antibody. Control is represented by HeLa cells transfected with pcDNA3.1 empty vector. (c) Co-IP of endogenous Beclin1 and PINK1 in SH-SY5Y cells stably expressing PINK-FL. IP as in (b). Control is represented by untransfected SH-SY5Y cells. (d) Co-IP of overexpressed Beclin1 and different PINK1 constructs. IP was performed with rat anti-HA antibody. Control samples were: PINK1-FL incubated without anti-HA antibody (no ab), IP performed without sample (no sample) 
PINK1-FL but not with its cleaved isoforms or the truncated PINK1 ${ }^{\text {W437X }}$ mutant in vivo. Our data suggest that the ability of PINK1 to bind Beclin1 is influenced by the lack of both the $\mathrm{N}$ - and $\mathrm{C}$-terminal domains but not by its kinase activity.
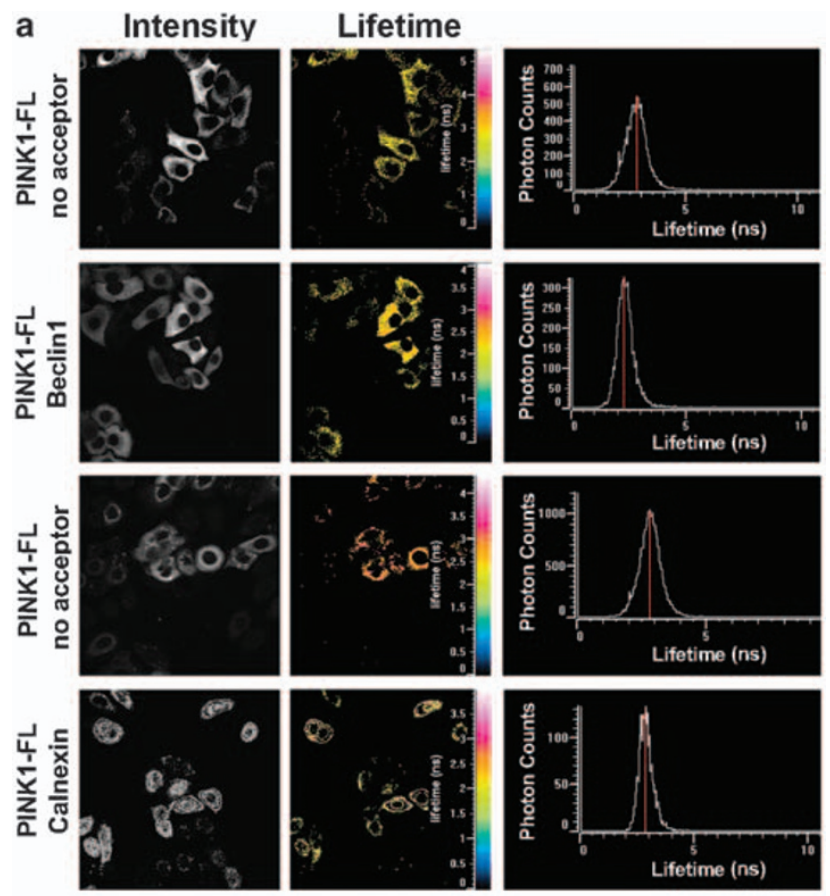

b

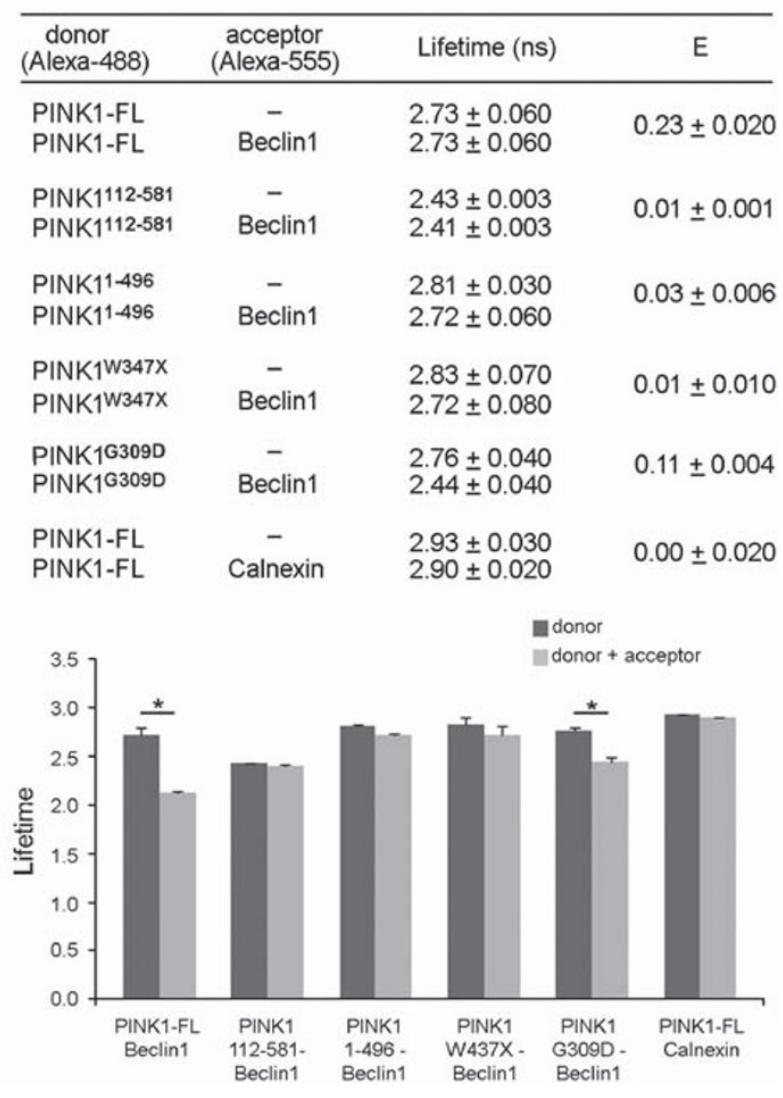

Both PINK1 and Beclin1 localize to the mitochondrial compartment. To assess the extent of co-localization of PINK1 and Beclin1, we transiently co-transfected PINK1-FL and Beclin1 in different cell types. Confocal microscopy analysis indicated evident co-localization of the two proteins both in HeLa and SH-SY5Y cells, which did not change after starvation, a potent inducer of the autophagic response (Figure 4a and Supplementary Figure 1). On testing PINK1deleted and mutant constructs in HeLa cells, co-localization with Beclin1 did not significantly change for PINK1 $1^{112-581}$ or PINK1 ${ }^{\text {G309D }}$, whereas it appeared to be variably reduced for $P I N K 1^{1-496}$ or PINK1 ${ }^{\text {W437X }}$. It should be noted that the overlap coefficient for Beclin1 and PINK $1^{112-581}$ is likely to be overestimated because of the diffuse localization of both proteins (Figure 4b).

We next asked where the interaction between PINK1 and Beclin1 could take place within the cell. By performing confocal microscopy experiments with organelle-specific markers, we confirmed that PINK1 localizes mainly to the mitochondria, with only a small proportion of it localizing to other compartments ${ }^{12,28}$ (Figure $4 \mathrm{c}$ and Supplementary Figure 2a). Mitochondrial localization did not significantly change for PINK $1^{1-496}$, PINK1 $1^{\mathrm{W} 437 \mathrm{X}}$ and PINK1 ${ }^{\mathrm{G} 309 \mathrm{D}}$, whereas PINK1 ${ }^{112-581}$ presented diffuse cytoplasmic localization, as expected (Figure 4e). In previous studies, Beclin1 has been detected within mitochondria, ER and the transGolgi network. ${ }^{29}$ We observed that Beclin1 localized considerably to mitochondria and, in lesser proportion, to the ER, whereas we failed to identify co-localization with Golgi or lysosome markers (Figure 4d and Supplementary Figure 2b). These findings indicate mitochondria as the only subcellular compartment in which both proteins abundantly localize, although they also seem to have partial cytosolic localization.

To assess whether PINK1 could influence the mitochondrial distribution of endogenous Beclin1, we performed subfractionation experiments on SH-SY5Y cells stably overexpressing PINK1-FL or PINK1 ${ }^{\text {W437X }}$ ability of which to bind Beclin1 is impaired. We were able to detect endogenous Beclin1 in both mitochondrial and cytoplasmic/microsomal fractions, with no significant differences between the two cell lines, as well as in a control cell line. Similarly, we observed no differences in PINK1-FL distribution when Beclin1 was either overexpressed or silenced (Figure 4f). Beclin1 distribution also did not change when PINK1-FL or PINK $1^{\text {W437X }}$ were transiently co-transfected with Beclin1 or when Beclin1 was expressed alone (data not shown). Thus, it seems that the

Figure 3 FLIM analysis of PINK1 and Beclin1 in HeLa cells. (a) Cells coexpressing HA-tagged PINK1 and Myc-tagged Beclin1 were labeled with donor fluorophore Alexa-488 (to label PINK1) \pm acceptor fluorophore Alexa-555 (to label Beclin1). PINK1-FL in the absence or presence of Calnexin labeling was used as control for negative interaction. The intensity images (left) show the standard immunostaining pattern for PINK1-FL in the absence or presence of Beclin1 or Calnexin staining. Corresponding FLIM images using a pseudocolor scale (middle) and relative graphs (right) visualize donor fluorophore lifetime $(n=3$ experiments, see Materials and Methods section). (b) Means \pm S.E.M. values and relative plotting of FLIM analysis of different PINK1 constructs, in several randomly selected cells. E, FRET efficiency. ${ }^{*} P<0.001$ 

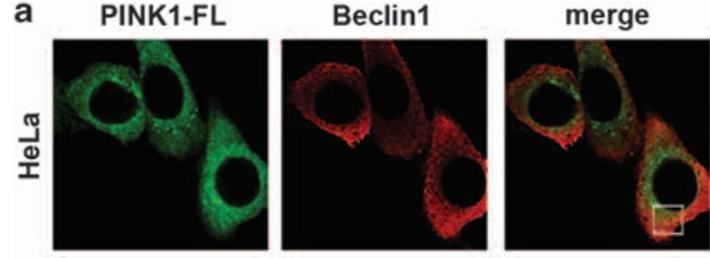

$R=0.56$
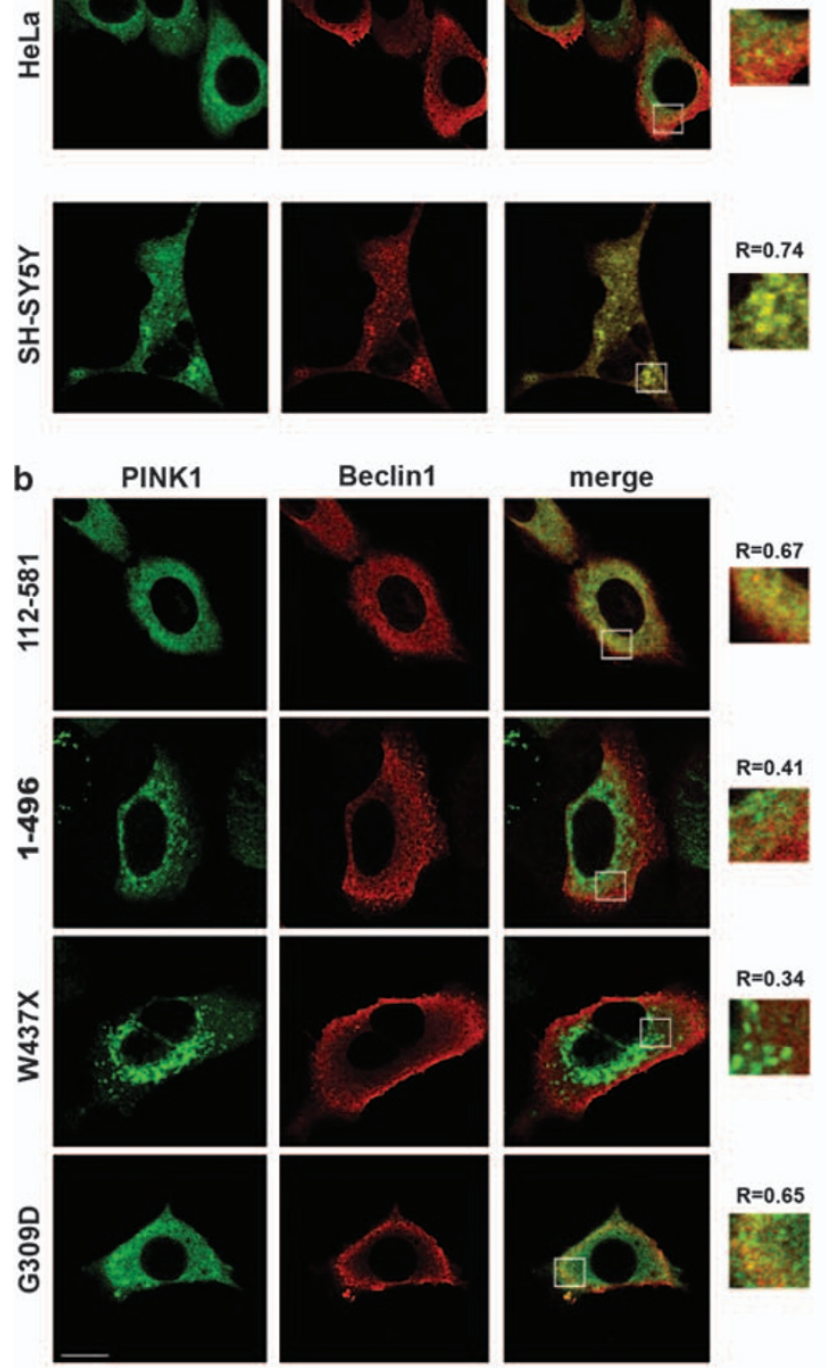

f Stable SH-SY5Y

Stable SH-SY5Y

Stable SH-SY5Y pcDNA

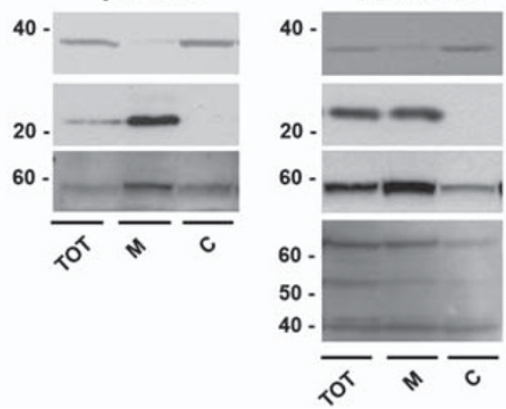

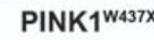

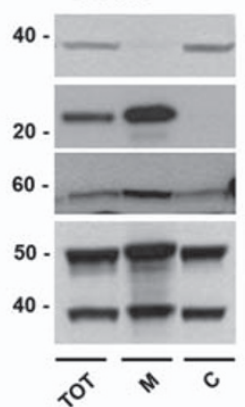

C

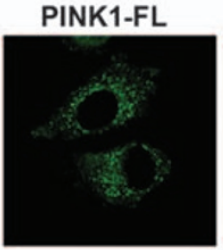

d
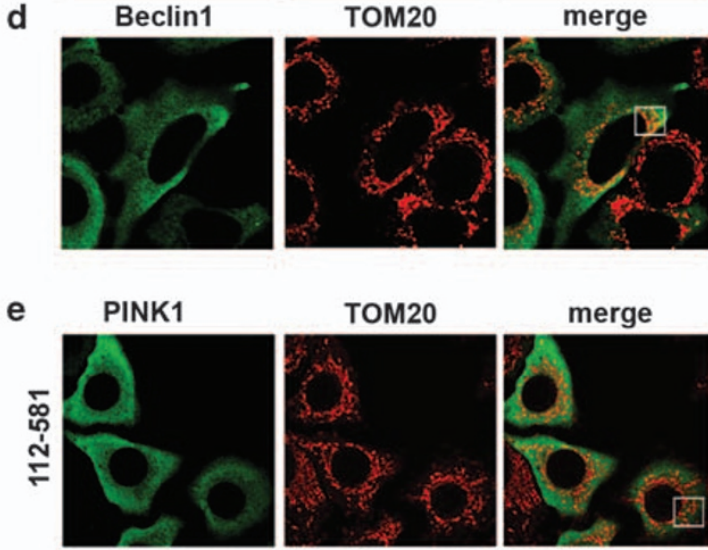

$\mathbf{R}=\mathbf{0 . 0 2}$
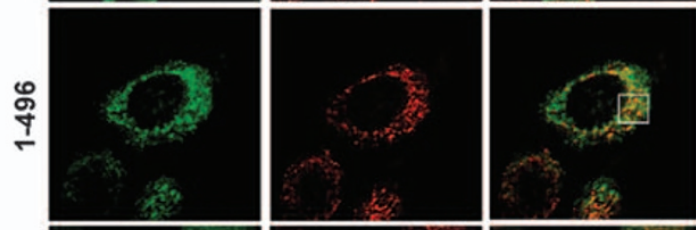

$R=0.66$
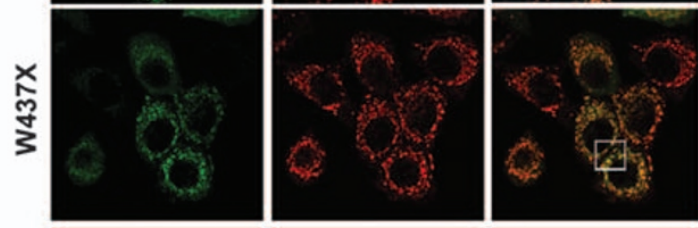

$\mathrm{R}=0.74$
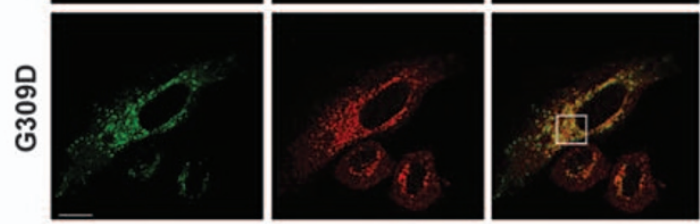

$\mathbf{R}=\mathbf{0 . 6 3}$

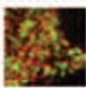

Figure 4 Co-localization and mitochondrial distribution of PINK1 and Beclin1. (a) Confocal images of HeLa cells and SH-SY5Y cells co-transfected with PINK1-FL and Beclin1. (b) HeLa cells co-transfected with different PINK1 constructs and Beclin1. (c-e) Confocal images of transfected HeLa cells representing co-localization of PINK1-FL (c), Beclin1 (d) and different PINK1 constructs (e) with the mitochondrial marker TOM20. Merge pictures and relative insets reveal co-localization. $R$ values represent the overlap coefficients. Scale bars: $10 \mu \mathrm{m}$. HA-tagged PINK1 constructs and myc-tagged Beclin1 were detected with anti-HA and anti-myc antibodies, respectively. (f) Western blotting analysis of subcellular localization of Beclin1, PINK1-FL or PINK1 ${ }^{\text {W437X }}$ in the following SH-SY5Y cells (from left to right): control cells stably expressing pcDNA; cells stably expressing HA-PINK1-FL or PINK1 ${ }^{\text {W437X. }}$; cells stably expressing HA-PINK1-FL and transfected with Beclin1 or with Beclin1 siRNA. PINK1 and Beclin1 were detected with anti-HA and anti-Beclin1 antibodies, respectively. Tot, Total Iysate; M, mitochondrial-enriched fraction; C, cytoplasmic/microsomal-enriched fraction. TIM23 and GAPDH were used as mitochondrial and cytoplasmic markers, respectively. 
interaction between PINK1 and Beclin1 is not relevant for the mitochondrial distribution of both proteins.

PINK1-FL, but not pathogenic mutant PINK1 ${ }^{\text {W437X }}$ enhances autophagy. As the main role of Beclin1 is to promote autophagosome formation and maturation, ${ }^{29,30}$ we reasoned that PINK1 could interact with Beclin1 to modulate autophagy. Two complementary strategies, based on immunofluorescence and western blotting, were optimized to detect autophagy. We first transiently overexpressed PINK1-FL in SH-SY5Y cells stably expressing GFP-LC3 under nutrient-rich and starvation conditions, and compared the number of PINK1-expressing and non-expressing cells that were positive for GFP-LC3 vacuoles, indicative of activated autophagy (Figure $5 \mathrm{a}$ ). In nutrient-rich conditions, PINK1-FL overexpression induced a nearly three-fold increase in the number of vacuole-positive cells compared with untransfected cells. Under starvation, the number of autophagic untransfected cells increased about three times compared with basal condition, and PINK1-FL overexpression produced a further significant increase of autophagic cells compared with starved control (Figure 5b). Notably, we did not observe any co-localization of PINK1 with the GFP-LC3 dots under nutrient-rich and starvation conditions (Supplementary Figure 3).

To confirm the ability of PINK1 to enhance autophagy, we monitored the conversion of endogenous LC3 from the cytoplasmic (LC3-I, $18 \mathrm{kDa}$ ) to the membrane-bound form (LC3-II, 16 kDa) in SH-SY5Y cells stably expressing PINK1. In this system, we quantified autophagy by assessing the LC3-II/ LC3-I ratio, as we observed that, in SH-SY5Y cells, autophagy activation was variably associated with LC3-II increase and/or LC3-I decrease. ${ }^{31}$ Under both nutrient-rich and starvation conditions, PINK1-FL cells showed a significant increase of the LC3-II/LC3-I ratio compared with control cells. Activation of autophagy was further confirmed by showing reduction of P62 levels in cells expressing PINK1 versus controls (Figure 5c).

To explore whether the observed results actually represented an increase of autophagy, we treated cells with the lysosomal protease inhibitor ammonium chloride, $\left(\mathrm{NH}_{4} \mathrm{Cl}\right)$, that prevents the degradation of autophagosomes. Immunofluorescence analysis showed that GFP-LC3 punctae dramatically increased under starvation conditions. In western blotting, treatment with $\mathrm{NH}_{4} \mathrm{Cl}$ induced an evident accumulation of LC3-II in both cell lines (Figure $5 \mathrm{~d}$ ). These findings suggest that PINK1-induced autophagy results from a true enhancement of the autophagic flux and not from the blockage of autophagic degradation. ${ }^{32}$

We next knocked down PINK1 expression using RNA in GFP-LC3 and wild-type SH-SY5Y cells, obtaining 60 and $80 \%$ decline of PINK1 mRNA expression, respectively. In immunofluorescence experiments, PINK1 silencing induced a significant decrease of starvation-induced autophagy when compared with control cells, which was confirmed by a reduction of the LC3-II/LC3-I ratio in western blotting (Figures $5 e$ and f).

To assess whether PINK1-related autophagy is mediated by the Beclin1-Vps34 complex, we first evaluated GFP-LC3 vacuole-positive cells in presence of 3-methyladenine (3MA), a compound known to block autophagy by inhibiting the activity of class III PI3K-Vps34, ${ }^{33}$ a Beclin1 effector that regulates autophagosome formation and maturation. ${ }^{30} \mathrm{We}$ showed that, in PINK1-expressing cells, increasing concentrations of 3MA were able to significantly decrease basal and starvation-induced autophagy (Figures $5 \mathrm{~g}$ and $\mathrm{h}$ ). To further verify the direct involvement of Beclin1, we then knocked down Beclin1 by siRNA. Beclin1 silencing also induced a significant reduction of autophagy in cells overexpressing PINK1, although the proportion of autophagic cells remained higher than that of control cells (Figures $5 \mathrm{i}$ and $\mathrm{j}$ ).

To assess the effect of distinct mutations on PINK1-related autophagy, we next monitored the proportion of autophagic cells and endogenous LC3 conversion in SH-SY5Y cells expressing PINK1-FL and either the W437X or the G309D mutant.

In immunofluorescence, the percentage of vacuole-positive cells was significantly lower in cells expressing PINK1 ${ }^{\text {W437X }}$ than those expressing PINK1-FL in nutrient-rich conditions, and overall comparable with untransfected controls under starvation (Figures 6a and c). Conversely, overexpression of PINK1 ${ }^{\text {G309D }}$ resulted in an increase in the number of autophagic cells that did not significantly differ from PINK1FL both under nutrient-rich and starvation conditions (Figures $6 b$ and $d)$. Similar results were obtained with LC3 western blotting (Figures $6 e$ and $f$ ).

Taken together, our data show that PINK1-FL is able to significantly enhance basal and starvation-induced autophagy, and that this effect is at least partly mediated by Beclin1. The PINK1-mediated autophagy is greatly reduced in presence of PINK1 ${ }^{\text {W437X }}$, ability of which to bind Beclin1 is largely impaired, but not in presence of PINK $1^{\mathrm{G} 309 \mathrm{D}}$, a protein with defective kinase activity but unaltered Beclin1 binding.

PINK1 overexpression does not affect mitochondrial morphology. We next sought to assess whether the observed effects of PINK1 on autophagy could be mediated by its activity on mitochondrial dynamics.

Mitochondrial morphology was analyzed in HeLa cells by co-transfecting a mitochondrially targeted yellow fluorescent protein (mtYFP) with mitofusin1 and DRP1 as positive controls for the induction of fusion or fission, and with either PINK1-FL, PINK1 $1^{\text {W437X }}$ or PINK1 ${ }^{\text {G309D }}$ and performing live imaging using confocal microscopy. For quantitative analysis of mitochondrial morphology, we distinguished between cells with tubular, intermediate or fragmented mitochondria, as described previously. ${ }^{34}$ Co-transfection of mtYFP with each construct was confirmed by immunofluorescence analysis (data not shown).

Live imaging of cells overexpressing mitofusin1 showed a higher number of cells with tubular mitochondria than mock-transfected cells, whereas the expression of DRP1 resulted in a significant increase of fragmented mitochondria. Transfection of mtYFP with PINK1-FL or mutants did not result in visible morphological changes of the mitochondrial network in comparison with control cells (Figure 7), suggesting that, at least in this experimental setting, PINK1 is not directly involved in the regulation of mitochondrial dynamics. 


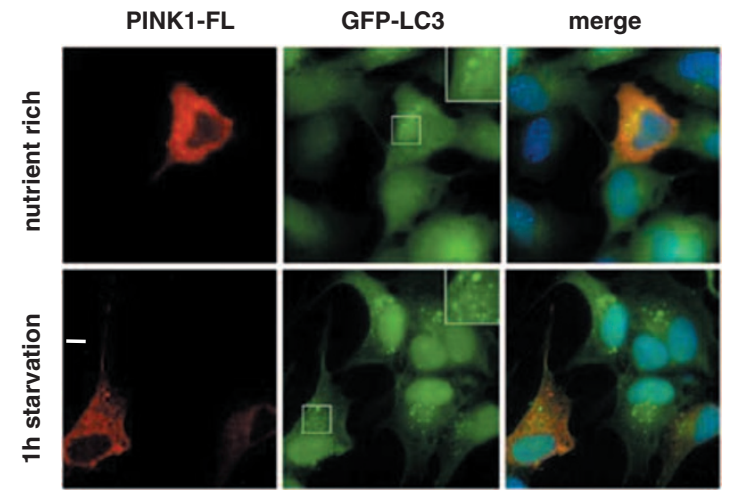

C
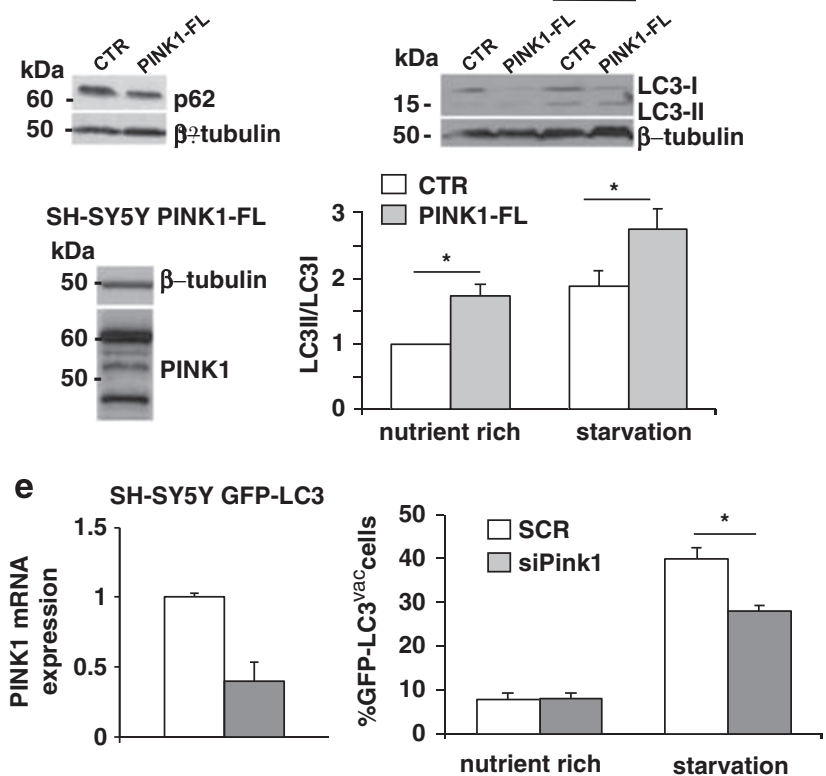

STARV

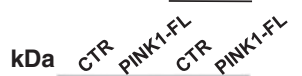

$12-12$ LC3-I

$15-\square$ LC3-II

50 - $\beta$-tubulin

$\square$ CTR

nutrient rich starvation

b

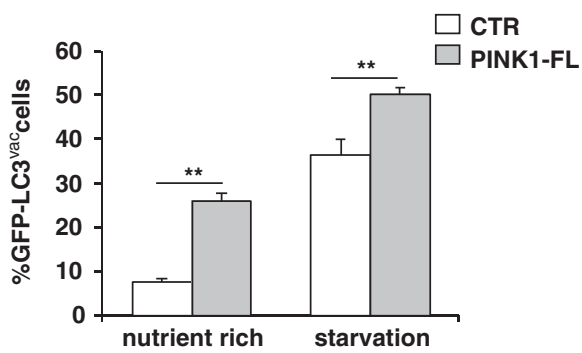

d
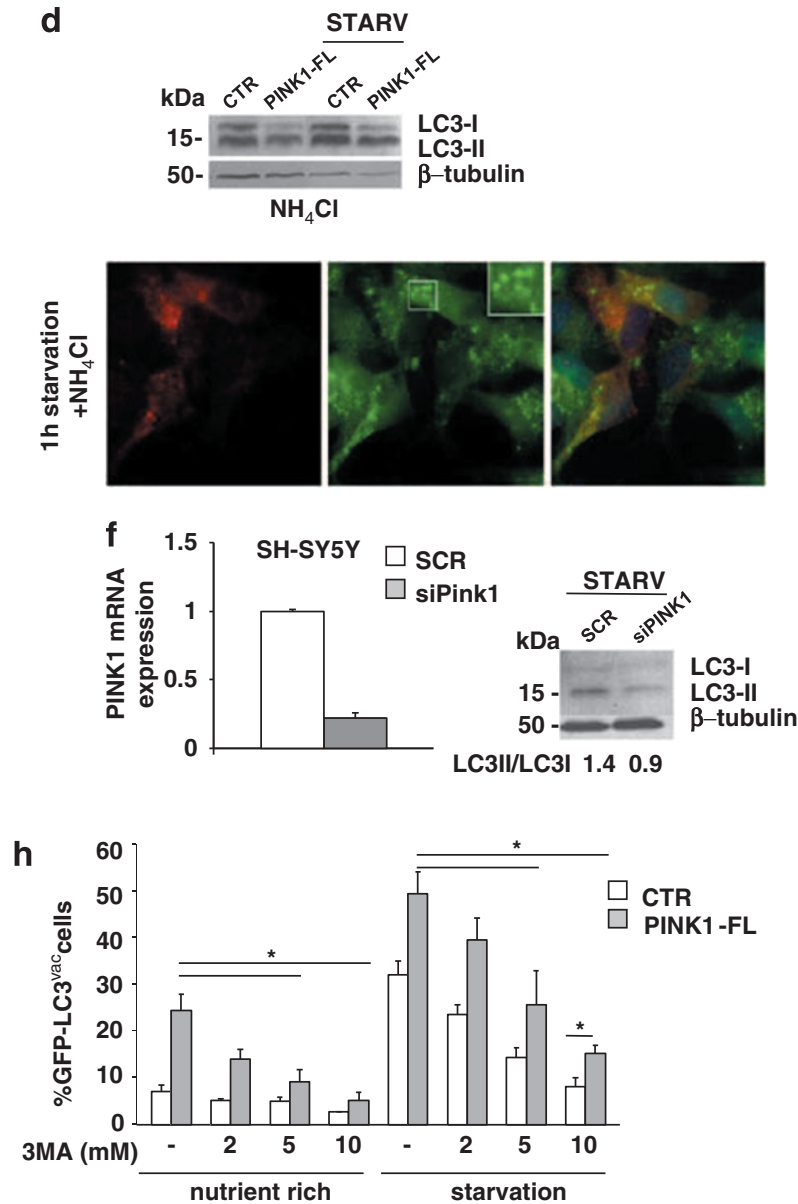

i

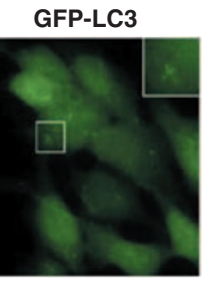

merge
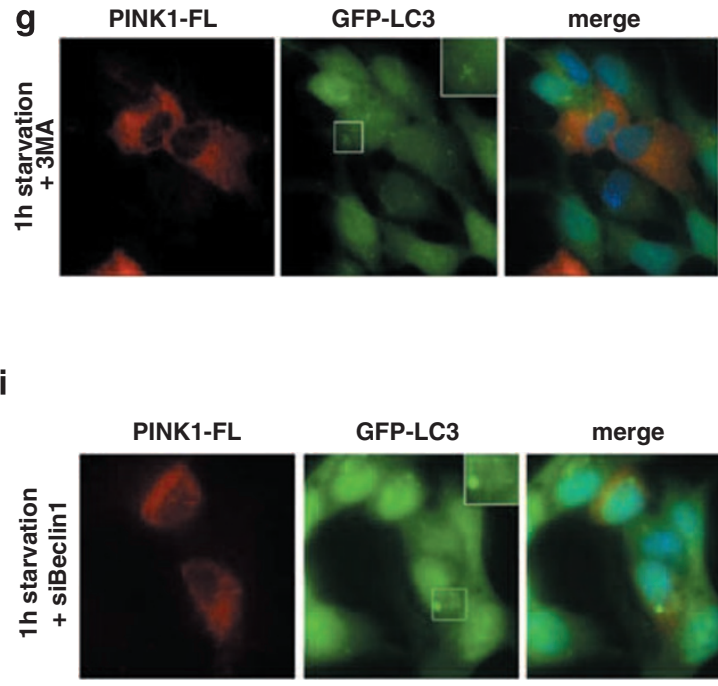

GFP-LC3

merge

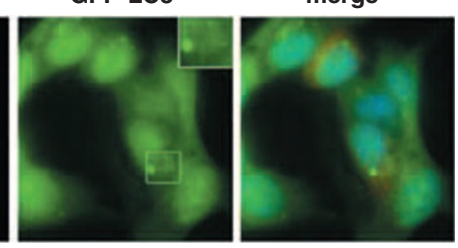

j

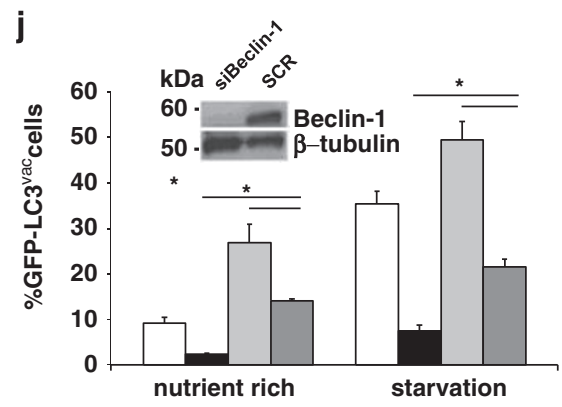

SCR+

empty vector

siBeclin1

+empty vector

SCR+

$\square$ PINK1-FL

siBeclin1+

PINK1-FL 


\section{Discussion}

In this study, we describe a new interaction between the pro-autophagic protein Beclin1 and PINK1, a protein mutated in autosomal recessive PD.

Beclin1, also known as ATG6, is a proautophagic protein that has been extensively studied as a tumor-suppressor factor and as an interactor of the anti-apoptotic protein $\mathrm{Bcl}-2 .^{29,35}$ Beclin1 expression decreases in an age-dependent manner in human brains, leading to a reduction of autophagy with aging, which possibly contributes to the progression of neurodegenerative diseases. ${ }^{36} \mathrm{~A}$ role of Beclin1 in neurodegeneration is also supported by the finding of reduced Beclin1 levels in affected brain regions of patients with Huntington's and Alzheimer's diseases; moreover, Beclin1 deficiency in cellular and animal models of these disorders was shown to induce disruption of neuronal autophagy, accumulation of mutant hungtintin and amyloid- $\beta$ and neuronal cell loss. ${ }^{36,37}$ Our data, to the best of our knowledge, correlate for the first time Beclin1 to a protein directly involved in PD pathogenesis, strengthening the link between autophagy and PD-related neurodegeneration.

Beclin1 is strictly modulated by the binding of Bcl-2 family members (Bcl-2/Bcl-XL), which results in autophagy inhibition. On receiving autophagic stimuli, this interaction is disrupted either by post-translational modifications (e.g. JNK-mediated Bcl-2 phosphorylation) or by competitive binding with $\mathrm{BH}$-only proteins, such as Bad, allowing activation of autophagy. ${ }^{35,38}$ Very recently, Zalckvar et al. ${ }^{39}$ have shown that death-associated protein kinase (DAPK)mediated phosphorylation of Beclin1 also promotes its dissociation from $\mathrm{Bcl}-\mathrm{XL}$ and triggers autophagy. Using this knowledge, it might be speculated that PINK1 could modulate autophagy either through direct phosphorylation of Beclin1 or its inhibitory interactors, or through competitive binding. In our study, we were not able to detect PINK1-mediated phosphorylation of Beclin1 in vitro. Moreover, kinase-defective PINK1 1 ${ }^{\text {G09D }}$, but not the truncated mutant PINK1 ${ }^{\text {W437X }}$, regulated autophagy with an efficiency similar to PINK1-FL, suggesting that this effect is mediated by Beclin1 binding and not by PINK1 kinase activity. The activation of autophagy by PINK1 was significantly reduced in the presence of Beclin1 silencing as well as exposure to 3MA, an autophagy blocker that inhibits Vps34, ${ }^{40}$ suggesting a direct involvement of PINK1 in the Beclin1-Vps34 macroautophagy pathway.
However we noted that, under both conditions, PINK1induced autophagy did not reach control cell levels, suggesting a possible role for PINK1 in distinct pathways. Indeed, recent studies have uncovered a number of Beclin1-dependent and -independent autophagy pathways, indicating that the macroautophagy process is far more complex than previously thought. $^{30,41,42}$ Further studies are needed to establish how PINK1 can interplay with Beclin1 multiple interactors and with other major autophagy effectors to trigger the autophagic response in neuronal cells.

In our study, PINK1 seems to positively regulate autophagy independently of its effects on mitochondrial dynamics. Indeed, in the presence of endogenous wild-type PINK1, neither overexpression of PINK1 nor of its mutants had a directly influence on mitochondria morphology, whereas it clearly affected basal and starvation-induced autophagy. These findings are in line with those previously reported for Parkin, which also seems to activate mitophagy in a way independent from its activity on mitochondrial morphology. ${ }^{20}$

After PINK1 silencing, we observed a significant reduction of starvation-induced autophagy in SH-SY5Y cells, whereas no obvious differences were evident in nutrient-rich condition. The apparent discrepancy with a previous study, in which PINK1 silencing was reported to indirectly enhance mitophagy through the activation of mitochondrial fission, ${ }^{19}$ could likely be explained by the different experimental settings adopted in the two studies, in particular the use of transient versus stable PINK1 silencing. In fact, it is plausible that a chronic reduction of PINK1 expression could activate compensatory pathways to counterbalance the damaging effects secondary to PINK1 deficiency, which are not yet appreciable at an early stage after silencing.

Interestingly, we observed that endogenous Beclin1 only immunoprecipitated PINK1-FL and that the interaction between PINK1 and Beclin1 was markedly reduced in the absence of the first 112 aa of PINK1. Taken together, these findings suggest that only PINK1-FL, and not the mature isoforms generated after mitochondrial import, is required to bind Beclin1. Indeed, previous data have shown a tight regulation of the relative amounts and subcellular distribution of these isoforms, implying distinct roles within cells. ${ }^{11,12}$

Both PINK1-FL and Beclin1 are found in the mitochondria and cytosol, making it difficult to exactly locate the subcellular compartment in which the two proteins interact. As Beclin1 does not possess a canonical amino-terminal targeting signal

Figure 5 Assessment of autophagy induction by PINK1-FL in SH-SY5Y cells. (a-c) Effect of PINK1-FL overexpression. (a) Representative pictures of SH-SY5Y cells stably expressing GFP-LC3 and transiently transfected with HA-tagged PINK1-FL. Appearance of GFP-LC3 autophagic vacuoles (GFP-LC3 ${ }^{\text {vac }}$ ) was assessed both under nutrient-rich condition and $1 \mathrm{~h}$ starvation. Merge pictures reveal co-localization. Insets show magnification of GFP-LC3 dots in PINK1-transfected cells. Scale bar $10 \mu \mathrm{m}$. (b) Mean percentage of untransfected versus PINK1-FL-transfected cells showing GFP-LC3 ${ }^{\text {vac }}(n=3$ experiments performed in quadruplicate; a minimum of 200 cells per experiment were analyzed; mean \pm S.E.M.). (c) Representative P62 blot (left) and LC3 blot (right) in SH-SY5Y cell lines stably expressing empty vector (control, CTR) versus PINK1-FL in nutrient-rich or starvation conditions (STARV). Expression of HA-tagged PINK1-FL is shown below. Densitometric analysis using LC3-II/LC3-I ratio is shown in the graph, measuring relative increments compared with the control sample in nutrient-rich condition, set at 1 (a minimum of 6 blots were analyzed for each condition; mean \pm S.E.M.). (d) Effect of treatment with $20 \mathrm{mM} \mathrm{NH}_{4} \mathrm{Cl}$. Representative picture of cells as in (a) after $1 \mathrm{~h}$ starvation, and representative LC3 blot as in (c), after treatment. $(\mathbf{e}, \mathbf{f})$ Effect of PINK1 silencing. (e) Quantitative real-time PCR showing reduction of PINK1 mRNA expression levels in GFP-LC3 SH-SY5Y cells and mean percentage of GFP-LC3 ${ }^{\text {vac }}$ cells in control (scramble: SCR) versus PINK1 silenced cells; (f) quantitative real-time PCR in SH-SY5Y cells and representative LC3 blot. (g and $\mathbf{h}$ ) Effect of treatment with 3-methyladenine (3MA). (g) Representative picture of cells as in (a) after treatment with 3MA $10 \mathrm{mM}$ and $1 \mathrm{~h}$ starvation; (h) Mean percentage of GFP-LC3 ${ }^{\text {vac }}$ cells as in (b), in absence or presence of increasing 3MA concentrations. (i and j) Effect of Beclin1 silencing. (i) Representative picture of cells as in (a) after Beclin1 silencing and $1 \mathrm{~h}$ starvation; (j) Mean percentage of GFP-LC3 ${ }^{\text {vac }}$ cells treated with either Beclin1 siRNA (siBeclin1) or scramble siRNA (SCR) and transfected with either pcDNA3.1 empty vector or PINK1-FL. Western blotting of Beclin1 after siRNA treatment is shown on the right. Statistical significance: ${ }^{*} P<0.01 ;{ }^{* *} P \leqslant 0.0001$ 
a
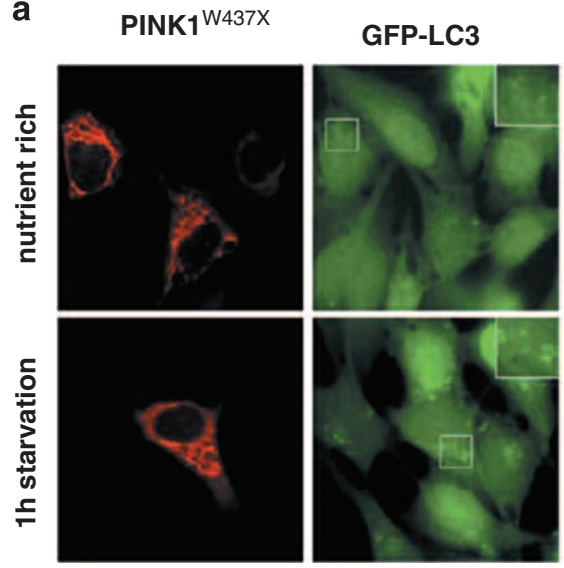

c

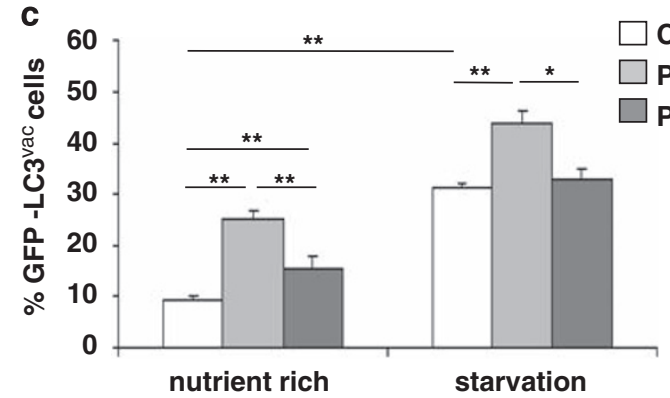

e
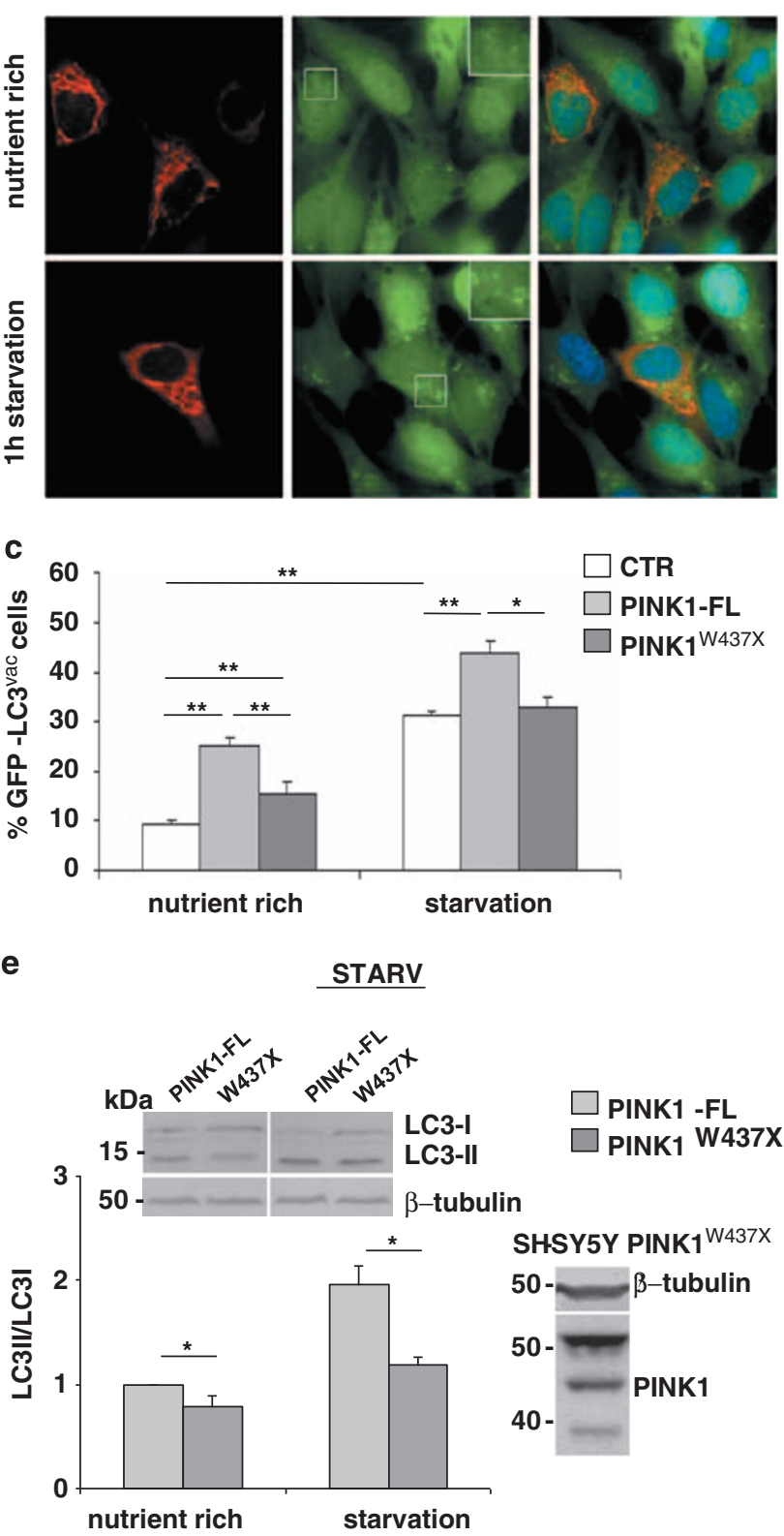

b
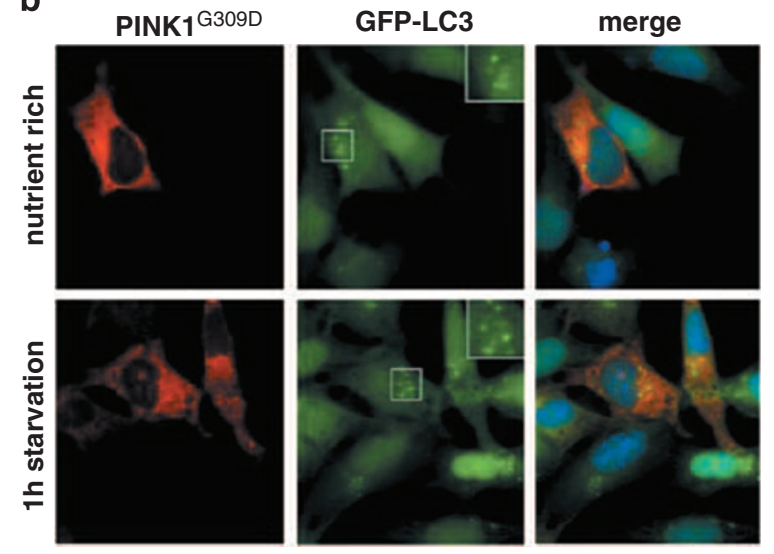

d

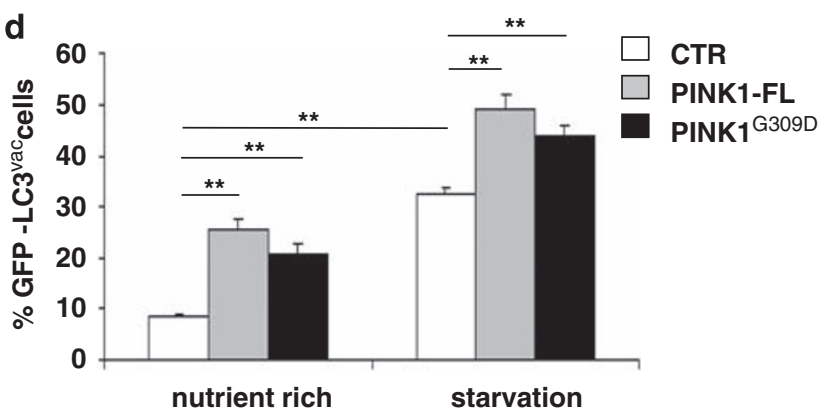

f

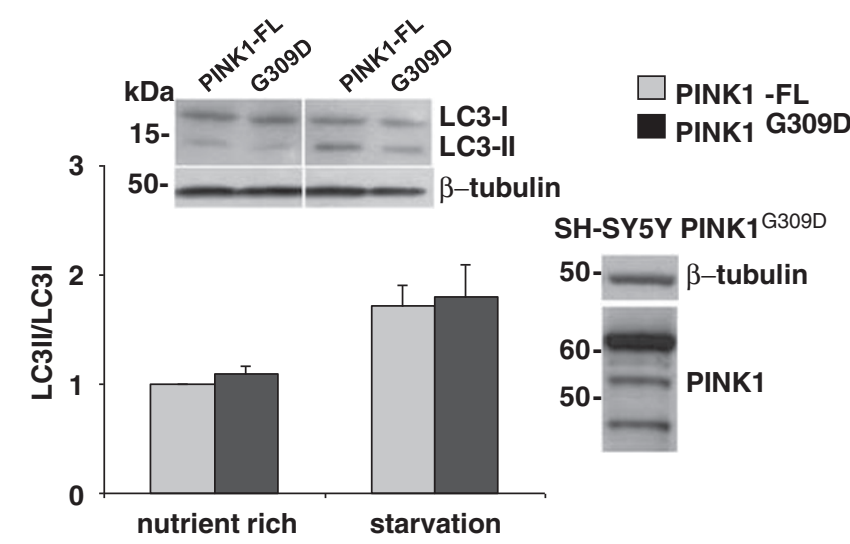

Figure 6 Effect of distinct mutations on PINK1-mediated autophagy. (a and $\mathbf{b})$ Representative pictures of SH-SY5Y cells stably expressing GFP-LC3 and transiently transfected with either HA-tagged PINK1 ${ }^{\mathrm{W} 437 \mathrm{X}}$ (a) or PINK1 $1^{\mathrm{G} 309 \mathrm{D}}$ (b). Appearance of GFP-LC3 ${ }^{\mathrm{vac}}$ was assessed both under nutrient-rich condition and $1 \mathrm{~h}$ starvation. (c and d) Mean percentage of cells showing GFP-LC3 ${ }^{\mathrm{vac}}(n=3$ experiments performed in quadruplicate; a minimum of 200 cells per experiment were analyzed; mean \pm S.E.M.). (e and f) Representative LC3 blots and densitometric analysis using LC3-II/LC3-I ratio of SH-SY5Y cells stably expressing PINK1-FL and PINK ${ }^{\text {W437X }}$ (e) or PINK ${ }^{\mathrm{G} 309 \mathrm{D}}$ (f). Relative increments were compared with the PINK1-FL sample in nutrient-rich condition, set at 1. Expression of each HA-tagged PINK1 mutant is shown on the right of each graph. Statistical significance: ${ }^{*} P<0.01 ;{ }^{* \star} P \leqslant 0.0001$.

for mitochondrial import, it can be hypothesized that it may be partly associated with the outer mitochondrial membrane (OMM) in which the interaction with PINK1 could take place. Other Beclin1-interacting proteins, such as Bcl-2 and Bcl-XL, are known to partly localize at the OMM through their C-terminal hydrophobic domain, and a recently published protease protection experiment on isolated mitochondria demonstrated a PINK1 immunoreactivity pattern similar to $\mathrm{Bcl}-2$. This study suggested that the $\mathrm{N}$-terminus of PINK1 would be inserted in the OMM through the putative transmem- brane domain (aa 94-110), with the kinase domain facing the cytosol. ${ }^{13}$ Such a localization would argue against our hypothesis, as both $\mathrm{N}$ - and C-terminal domains of PINK1 are required to ensure a strong Beclin1 binding. Nevertheless, it is reasonable to speculate that a pool of PINK1-FL would not enter the OMM, but remain associated with mitochondria through anchoring proteins. In line with this hypothesis, Weihofen et al. ${ }^{12}$ have proposed that binding to Cdc37/ Hsp90 would destine PINK1 to mitochondrial import and processing, while in the absence of Hsp90, PINK1 would 


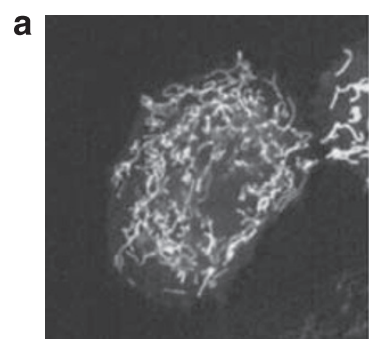

CTR

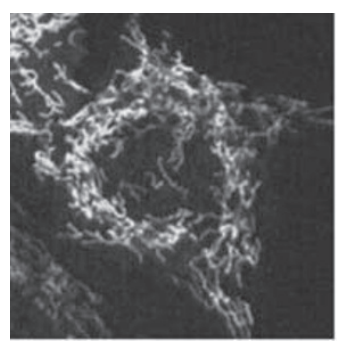

PINK1-FL

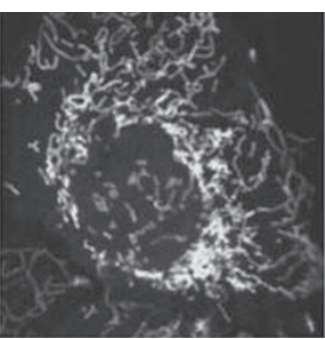

Mfn1

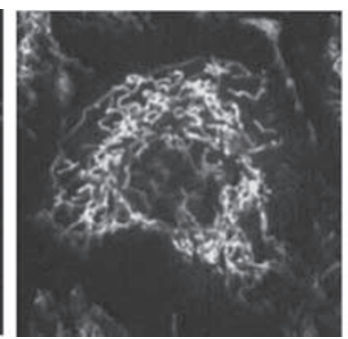

PINK1 W437X

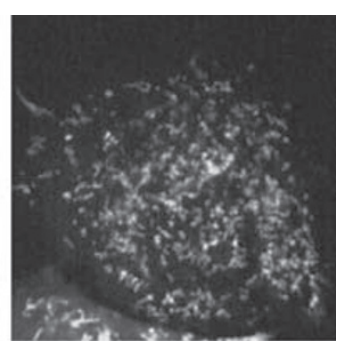

DRP1

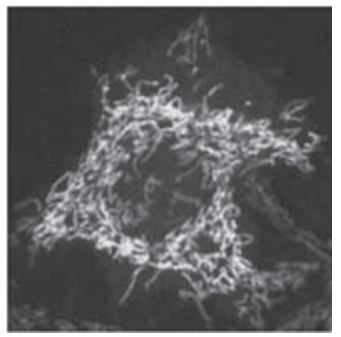

PINK1 ${ }^{\text {G309D }}$

b

tubular

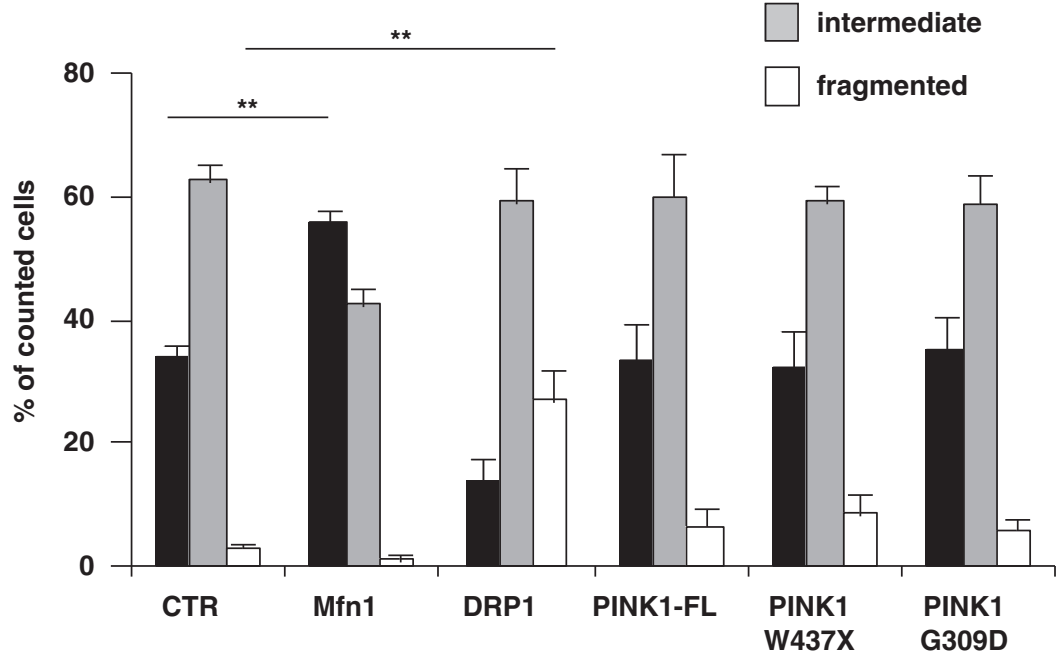

Figure 7 Analysis of mitochondrial morphology in HeLa cells expressing PINK1 constructs. (a) HeLa cells were co-transfected with mtYFP and empty vector (mock) or the indicated proteins. Representative confocal images of mtYFP fluorescence from randomly selected cells are shown. Scale bar: $10 \mu \mathrm{m}$. (b) Morphometric analysis of mitochondrial shape. A total of 30 randomly selected images of mtYFP fluorescence were acquired, stored and classified as described. Data represent mean \pm S.D. of at least three independent experiments. Mfn1: mitofusin 1. Statistical significance: ${ }^{*} P<0.001$.

remain attached to mitochondria as a full-length precursor. More recently, the same authors reported a new interaction of PINK1 with Miro and Milton, two proteins that are part of a complex connecting mitochondria to microtubules. Only Miro spans the OMM with its C-terminus, and thereby can anchor the cytosolic protein Milton and other members of the complex, such as PINK1, to the mitochondrion. ${ }^{27}$ Interestingly, overexpressions of Miro and Milton were shown to induce accumulation of PINK1-FL in the mitochondrial fraction, suggesting the existence of a regulatory mechanism controlling the pool of PINK1-FL, which is required to act at the mitochondrial outer membrane.

In conclusion we show that, besides its many protective functions, PINK1 may also have a relevant role in promoting basal and starvation-induced autophagy. The identification of autophagy as a key protective mechanism against neuronal cell loss has represented a crucial step toward the development of new therapeutic strategies for neurodegeneration, ${ }^{23}$ and autophagy-inducing drugs have been shown to bear neuroprotective activity in cellular and animal models of neurodegenerative diseases. ${ }^{24}$ Using this knowledge our findings represent a major progress in the current understanding of PD pathogenesis, and open new exciting research perspectives.

\section{Materials and Methods}

Yeast-two-hybrid analysis. A GAL4-based yeast two-hybrid screening was performed as described previously. ${ }^{43}$ Three PINK1 baits (N-terminus, aa 1-100; kinase domain and C-terminus, aa 110-581; full-length protein, aa 1-581) were cloned in pGBD-B vector (kind gift of Prof. David Markie, University of Otago, 
New Zealand) by gap repair, and used to screen a pre-transformed human brain cDNA library (Human Brain BD Matchmaker, BD Biosciences, San Jose, CA). Potential interactions were checked for the activation of two reporter genes (HIS3 and $A D E 2)$ by growth on selective medium. Specificity and reproducibility of interactions were further confirmed by re-cloning interacting preys in PACT2-B vector and mating them with the original bait strain and with an unrelated bait strain ( $\beta 2$-microglobulin, aa 30-96). For positive clones, interaction sequence tags were determined by sequencing the PCR fragments obtained from prey inserts and the corresponding genes were identified by bioinformatic search of nucleic acid databases.

Eukaryotic expression vectors. The PINK1 constructs were all tagged at $\mathrm{C}$-terminus with HA epitope. The constructs PINK1-FL, PINK1 ${ }^{\text {W437X }}$ and PINK1 $1^{\mathrm{G} 309 \mathrm{D}}$ have been described previously. ${ }^{28}$ The PINK $1^{1-496}$ and PINK1 ${ }^{112-581}$ constructs were generated from PINK1-FL cDNA by introducing, respectively, an EcoRI restriction site at position 497 and a start codon at position 112 using the QuickChange II XL Site-Directed Mutagenesis Kit (Stratagene, La Jolla, CA, USA). The two fragments, 1-496 and 112-581, were then subcloned in pcDNA 3.1 + +) (Invitrogen, Carlsbad, CA, USA) and HA-tagged at the C-terminus as described. ${ }^{28}$ The Beclin1 CDNA omitting the stop codon was amplified from human cDNA using primers $5^{\prime}$-CCCAAG CTTGGGATGGAAGGGTCTAAGAC-3' (sense; Hindll tail) and $5^{\prime}$-GGAATTCTTT GTTATAAAATTGTGAGGACAC- $3^{\prime}$ (antisense; EcoRI tail) and cloned into the Hindll and EcoRl sites of pcDNA3.1 $(+)$. The Myc tag was introduced at the C-terminus and the stop codon was inserted into sequence. The pEGFPC1-LC3 plasmid was a kind gift from Dr. Francesca De Marchi (International Centre for Genetic Engineering and Biotechnology, Trieste, Italy).

Human PINK1 shRNA expression plasmid was constructed using the pSilencer 3.1 H1-hygro system (Applied Biosystems, Foster City, CA, USA). Sense and antisense oligonucleotides were synthetized as previously described. ${ }^{12}$ The pSilencer hygro vector expressing a scramble shRNA was used as negative control.

Analysis of mitochondrial morphology was performed using pEYFP-Mito (Clontech, Carlsbad, CA, USA), human mitofusin1 (pCB6Myc-MFN1) and human DRP1 (pcDNA3.1-DRP1) plasmids, kindly provided by Prof. Luca Scorrano (University of Geneva Medical School, Geneva, Switzerland and Dulbecco-Telethon Institute, Padua, Italy).

Cell cultures, stable expression and treatments. Human HeLa and SH-SY5Y cells were cultured in Dulbecco's modified Eagle's medium (DMEM; Invitrogen), supplemented with $2 \mathrm{mM}$ L-glutamine, $200 \mathrm{U} / \mathrm{ml}$ penicillin, $200 \mathrm{mg} / \mathrm{ml}$ streptomycin, $1 \mathrm{mM}$ sodium pyruvate and $10 \%$ heat inactivated FBS at $37^{\circ} \mathrm{C}$ in $95 \%$ humidifier air and $5 \% \mathrm{CO}_{2}$. For serum and amino-acid deprivation studies (starvation), cells were cultured in serum-free Earle's Balanced Salt Solutions medium (EBSS; Invitrogen) at $37^{\circ} \mathrm{C}$ for $1 \mathrm{~h}$ in the presence or absence of $20 \mathrm{mM}$ $\mathrm{NH}_{4} \mathrm{Cl}$ or $3 \mathrm{MA}$ at concentrations of $2 \mathrm{mM}, 5 \mathrm{mM}$ and $10 \mathrm{mM}$ (Sigma Aldrich, St Louis, MO, USA).

Stable transfectants expressing PINK1-FL-HA, PINK1 ${ }^{\text {W437X }}-\mathrm{HA}, \mathrm{PINK} 1^{\mathrm{G} 309 \mathrm{D}}$ -HA and GFP-LC3 CDNA were obtained after transfection of respective expression vectors in SH-SY5Y human neuroblastoma cells. Transfected cells were cultured as bulk in a selective medium containing $1 \mathrm{mg} / \mathrm{ml} \mathrm{G418} \mathrm{(Invitrogen)} \mathrm{for} 20$ days, and then screened for protein expression by western blotting. As a control, a cell line stably expressing the pCDNA3.1 empty vector was also created following the same protocol.

To knock down endogenous PINK1 expression, wild-type and GFP-LC3 SHSY5Y cells were transfected with PINK1 or scramble shRNA expression vectors. After $72 \mathrm{~h}$, PINK1 mRNA expression was verified by quantitative real time PCR.

For Beclin1 knockdown, siRNA duplex oligoribonucleotides were purchased from Invitrogen (BECN1 Stealth Select RNAi; HSS112742). The Stealth RNAi Negative Control Duplex (Invitrogen) was used as negative control. The SH-SY5Y cells either stably expressing GFP-LC3 or PINK1-FL were transfected with 100 pmol siRNA, according to manufacturer's instructions. To increase Beclin1 silencing efficiency, siRNA transfection was repeated after $24 \mathrm{~h}$, co-transfecting PINK1-FL expression vector in GFP-LC3 SH-SY5Y cells. Beclin1 knockdown and PINK1 expression were checked $48 \mathrm{~h}$ from the second transfection by western blotting. All transfections have been performed using Lipofectamine 2000 transfection reagent (Invitrogen).

GST pull down. The GST -pull down was performed by standard techniques. The GST-PINK $1^{112-581}$ and GST-PINK $1^{112-496}$ constructs have been described previously. ${ }^{28}$ The GST-PINK1-FL, GST-PINK1 ${ }^{77-581}$ and GST-PINK $1^{77-496}$ constructs were generated from pcDNA3.1 PINK1-FL or pcDNA3.1 PINK1 ${ }^{1-496}$ by PCR using a sense oligonucleotide carrying the restriction site $B a m H I$ at position
1 and 77 of the corresponding plasmid and a antisense oligonucleotide, EcoRI, in frame with the GST protein. The GST-tagged constructs were expressed in the Escherichia coli strain BL21 and GST-PINK1 fusion proteins were produced under the induction by $0.1 \mathrm{mM}$ isopropyl thiogalactopyranoside (Sigma) for $3 \mathrm{~h}$ at room temperature. Recombinant proteins were purified by affinity absorption using glutathione-Sepharose 4B (Amersham Biosciences AB, Uppsala, Sweden) according to the manufacturer's instructions, and then incubated for $2 \mathrm{~h}$ at $40^{\circ} \mathrm{C}$ with lysates from HeLa cells expressing Beclin1-Myc followed by extensive washings. Pull-down products were separated by SDS-PAGE and analyzed by western blotting with anti-Myc antibody.

Q-RT-PCR. Total RNA was extracted using the RNeasy kit (Qiagen, Hilden, Germany) and then reverse transcribed with SuperScript II Reverse Transcriptase (Invitrogen). Resulting cDNAs were quantified by real-time PCR using SYBR green master mix (Applied Biosystems) on the HT-7900 platform (Applied Biosystems) using the following primers: PINK1-Fw: $5^{\prime}$-CAAGAGGCTCAGCTACCTGCAC-3'; PINK1-Rev: 5'-TGTCTCACGTCTGGAGGCACT-3'; GAPDH-Fw: 5'-CGCTTCGCT CTCTGCTCCT-3'; GAPDH-Rev: 5'-CCTTCACCTTCCCCATGGT-3'. The relative expression was calculated using the $\Delta \Delta C_{\mathrm{t}}$ method, and normalized to GAPDH expression.

Western blotting analysis. For western blotting analysis, $50 \mu \mathrm{g}$ of samples were subjected to SDS-PAGE and probed with the following antibodies: rat anti-HA (Roche Diagnostics, Indianapolis, IN, USA) or rabbit anti-HA (Sigma) for the detection of PINK1 proteins, goat anti-Myc or rabbit anti-Beclin1 (Novus Biologicals, Littleton, CO, USA) for Beclin1 protein, mouse anti-LC3 (MBL International, Woburn, MA, USA), mouse anti-P62 (BD Biosciences), mouse anti- $\beta$ tubulin (Sigma), rabbit anti-GAPDH (Santa Cruz Biotechnology, Santa Cruz, CA, USA) and mouse anti-TIM23 (BD Biosciences). All samples were normalized to $\beta$-tubulin.

Densitometric analysis of LC3 blots was performed using the software Quantity One 4.6.5 (Bio-Rad, Hercules, CA, USA). Activation of autophagy was evaluated using LC3-II/LC3-I ratio, measuring relative increments of each sample compared with the control sample in nutrient-rich condition, set at $1 .^{32}$

Co-immunoprecipitation assay. The HeLa cells were either transfected with vectors expressing PINK1-FL or co-transfected with vectors expressing PINK1FL or each PINK1 mutant and Beclin1. Both HeLa cells and SH-SY5Y cells stably expressing PINK1-FL were lysed in 1\% Triton X-100 buffer (10 mM HEPES (pH 7.5), $142.5 \mathrm{mM} \mathrm{KCl}, 5 \mathrm{mM} \mathrm{MgCl}, 1 \mathrm{mM}$ EDTA), containing protease and phosphatase inhibitor cocktails (Pierce, Rockford, IL, USA). Total cell extracts were centrifuged at $6000 \times g$ for $15 \mathrm{~min}$.

To immunoprecipitate endogenous Beclin1, $1 \mathrm{mg}$ of the lysate was incubated overnight at $4{ }^{\circ} \mathrm{C}$ with $4 \mu \mathrm{g}$ of rabbit polyclonal anti-Beclin1 antibody (Novus) and protein A Agarose (Roche). To immunoprecipitate PINK1, $500 \mu \mathrm{g}$ of the lysates were incubated overnight at $4{ }^{\circ} \mathrm{C}$ with $1 \mu \mathrm{g}$ of rat monoclonal anti-HA antibody (Roche) and protein $\mathrm{G}$ Sepharose (Roche). The immune complexes were then washed three times with lysis buffer without Triton $X-100$ by centrifugation. Samples were heated in SDS sample buffer and processed by western blotting.

Immunofluorescence and confocal microscopy. Immunofluorescence analysis was performed as described previously ${ }^{28}$ using the following antibodies: anti-HA (Covance, Berkeley, CA, USA) to detect PINK1 proteins, anti-Myc (Novus Biologicals) to detect Beclin1, anti-Calnexin (Sigma), antiTOM20 (BD Biosciences), anti-LAMP1 (Developmental Studies Hybridoma Bank, lowa City, IA, USA), anti-Golgin (Molecular Probes, Eugene, OR, USA). Primary antibodies were visualized using the appropriate secondary antibodies conjugated with either Alexa Fluor 488 or Alexa Fluor 555 (Molecular Probes). All images were acquired using a confocal microscope (PCM Eclipse TE300, Nikon Instruments, Tokyo, Japan). Merged images were obtained by EZ2000 software. The Overlap Coefficient $(R)$ was calculated on several randomly selected cells from different slides, using WCIF ImageJ software (www.uhnresearch.ca/facilities/wcif/imagej/). For each co-localization showing an $R$ value $>0.30$, overlay masks are presented in Supplementary Figure 2c.

FRET-FLIM assay. The FRET-FLIM measurements rely on the observation that fluorescence lifetime of a donor fluorophore is shorter in close proximity $(<10 \mathrm{~nm})$ of an acceptor fluorophore, in which the energy transfer only depends on the distance of the two protein-interacting domains and not on their relative 
concentration. The FRET-FLIM assays were performed as previously described. Briefly, fixed HeLa cells transiently transfected with PINK1 constructs and Beclin1 were labeled with appropriate primary and secondary antibodies (see above). Samples were examined with a C1 confocal microscope attached to a TE2000 inverted microscope and equipped with a LIMO lifetime imaging system (Nikon). Cells were excited under two-photon excitation at $750 \mathrm{~nm}$ with a Ti:sapphire ultrafast laser source (Mai Tai Laser 750-850, Spectra Physics, CA, USA). Photons were accumulated for $60 \mathrm{~s}$ and data were analyzed using EZ LIMO Software 3.40 (Nikon). For each condition, a minimum of three experiments in duplicate were performed, analyzing 7-8 cells from each slide for statistical analysis. The FRET efficiency $(E)$ was calculated as: $E=(1-\tau \mathrm{Da} / \tau \mathrm{D})$ where $\tau \mathrm{D}$ and $\tau \mathrm{DA}$ are the lifetimes of the donor alone and the donor in the presence of acceptor, respectively. ${ }^{44}$

Subcellular fractionation. Mitochondrial and cytosolic subcellular fractions were isolated by cell disruption followed by differential centrifugation and washing as described previously, ${ }^{4}$ with some modifications. Briefly, homogenates were centrifuged at $750 \times \mathrm{g}$ for $10 \mathrm{~min}$ at $4^{\circ} \mathrm{C}$, and supernatants were then centrifuged at $10000 \times g$ for $15 \mathrm{~min}$ at $4{ }^{\circ} \mathrm{C}$. Pellets contained the enriched mitochondrial fraction, whereas supernatants contained the cytoplasmic/ microsomal fraction. Equal amounts of proteins from the two fractions were analyzed by western blotting. Beclin1 was detected with anti-Beclin1 antibody, whereas PINK1 was detected using rat monoclonal anti-HA antibody. Anti-TIM23 and anti-GAPDH antibodies were used as molecular markers of the two fractions.

Analysis of GFP-LC3 immunofluorescence. The SH-SY5Y cells stably expressing GFP-LC3 were transfected with HA-tagged PINK1-FL, PINK $1^{\text {W437X }}$ or PINK ${ }^{\text {G309D }}$, and immunostained with anti-HA antibody to mark PINK1-expressing cells. In each acquired field, GFP-LC3 cells not expressing PINK1 were used as controls. For PINK1 silencing, GFP-LC3 SH-SY5Y cells were either transfected with PINK1 or scramble shRNA expression plasmid. For Beclin1 silencing, GFP-LC3 SH-SY5Y cells were transfected with either HA-tagged PINK1FL or pcDNA3.1 with or without Beclin1 or scramble siRNA. Autophagy was quantified by counting the percentage of cells exhibiting the accumulation of GFP-LC3 vacuoles (GFP-LC3vac). Cells mostly presenting a diffuse distribution of GFP-LC3 were considered non-autophagic, whereas cells presenting several intense GFP-LC3 vacuoles were classified as autophagic. ${ }^{44}$ Experiments were performed in nutrient-rich condition and after starvation, in absence or presence of treatments. Optimal setting for starvation-induced autophagy was established in a 4-h time course experiment. Each experiment set was repeated at least three times in quadruplicate and a minimum of 200 cells per experiment were analyzed. Each GFP-LC3 staining was read by two independent investigators blinded to the experimental setting.

Mitochondrial morphology analysis by live imaging. Analysis of mitochondrial morphology was performed as described ${ }^{34}$ Cells were co-transfected with plasmids expressing EYFP-Mito and empty vector (control), regulators of mitochondrial dynamics (human Mitofusin1 or human DRP1) or PINK1 constructs (PINK1-FL, PINK1 $1^{\text {W437X }}$ or PINK1 ${ }^{\text {G309D }}$ ), using a 1:2 ratio. Confocal microscopy was performed using Perkin Elmer (Foster City, CA, USA) UltraVIEW Spinning Disk Confocal Microscope and EMCCD Hamamatsu C9100 imaging camera. This system is equipped with a stage incubator from OkoLab (www.okolab.com), allowing to work with live cells maintained under stable conditions of temperature, $\mathrm{CO}_{2}$ and humidity. A total of 30 randomly selected images of cells expressing mtYFP for each transfection were acquired. Morphometric analysis of mitochondrial network was performed examining 100-150 cells for each experimental condition. Cells were divided into three classes according to mitochondrial shape: tubular mitochondrial network, intermediate or fragmented mitochondrial structures. Experiments were repeated at least three times, and analysis was performed by three operators independently. Co-transfection efficiency was assessed performing in parallel live imaging and immunofluorescence analyses on the same samples.

Statistical analysis. Data are expressed as means \pm S.E.M. values. Student's $t$-test or ANOVA were used for calculation of $P$-values as appropriate. The distribution of values for each variable was tested for normality using Kolmogorov-Smirnov test.

\section{Conflict of interest}

The authors declare no conflict of interest.
Acknowledgements. We are grateful to Dr. Sylvia Lombardo for technical support, Dr. Alessandro Ferraris for statistical analysis support, Prof. David Markie for help in designing the yeast two-hybrid experiment and for providing the pGBD-B vector, Dr. Francesca De Marchi for providing GFP-LC3 (pEGFPC1-LC3) and Prof. Luca Scorrano for providing human Mitofusin1 (pCB6Myc-MFN1) and human WT DRP1 (pcDNA3.1-DRP1) plasmids. This study was supported with grants from Telethon Foundation (GGP07210 to EMV and GC), Italian Ministry of Health (Ricerca Corrente 2008-2009, Ricerca Finalizzata 2004 and 2006 and ex art.56 to $\mathrm{BD}$ ) and Fondazione Cariplo (to GC). The support of Fondazione Livio Patrizi and Transgenomics is also gratefully acknowledged. The sponsors had no role in study design, data collection and analysis, decision to publish or preparation of the paper.

1. Gasser T. Mendelian forms of Parkinson's disease. Biochim Biophys Acta 2009; 1792 : 587-596

2. Valente EM, Abou-Sleiman PM, Caputo V, Muqit MM, Harvey K, Gispert S et al. Hereditary early-onset Parkinson's disease caused by mutations in PINK1. Science 2004; 304 : $1158-1160$.

3. Tan EK, Skipper LM. Pathogenic mutations in Parkinson disease. Hum Mutat 2007; 28: 641-653

4. Petit A, Kawarai T, Paitel E, Sanjo N, Maj M, Scheid M et al. Wild-type PINK1 prevents basal and induced neuronal apoptosis, a protective effect abrogated by Parkinson diseaserelated mutations. J Biol Chem 2005; 280: 34025-34032.

5. Wang HL, Chou AH, Yeh TH, Li AH, Chen YL, Kuo YL et al. PINK1 mutants associated with recessive Parkinson's disease are defective in inhibiting mitochondrial release of cytochrome c. Neurobiol Dis 2007; 28: 216-226.

6. Wood-Kaczmar A, Gandhi S, Yao Z, Abramov AS, Milian EA, Keen G et al. PINK1 is necessary for long term survival and mitochondrial function in human dopaminergic neurons. PLOS ONE 2008; 3: e2455.

7. Deng H, Jankovic J, Guo Y, Xie W, Le W. Small interfering RNA targeting the PINK1 induces apoptosis in dopaminergic cells SH-SY5Y. Biochem Biophys Res Commun 2005; 337: 1133-1138.

8. Sandebring A, Thomas KJ, Beilina A, van der BM, Cleland MM, Ahmad R et al. Mitochondrial alterations in PINK1 deficient cells are influenced by calcineurin-dependent dephosphorylation of dynamin-related protein 1. PLOS ONE 2009; 4: e5701.

9. Pridgeon JW, Olzmann JA, Chin LS, Li L. PINK1 protects against oxidative stress by phosphorylating mitochondrial chaperone TRAP1. PLOS Biol 2007; 5: e172.

10. Haque ME, Thomas KJ, D'Souza C, Callaghan S, Kitada T, Slack RS et al. Cytoplasmic Pink1 activity protects neurons from dopaminergic neurotoxin MPTP. Proc Natl Acad Sci USA 2008; 105: 1716-1721

11. Lin W, Kang UJ. Characterization of PINK1 processing, stability, and subcellular localization. J Neurochem 2008; 106: 464-474.

12. Weihofen A, Ostaszewski B, Minami Y, Selkoe DJ. Pink1 Parkinson mutations, the Cdc37/ Hsp90 chaperones and Parkin all influence the maturation or subcellular distribution of Pink1. Hum Mol Genet 2008; 17: 602-616.

13. Zhou C, Huang Y, Shao Y, May J, Prou D, Perier C et al. The kinase domain of mitochondrial PINK1 faces the cytoplasm. Proc Natl Acad Sci USA 2008; 105: 12022-12027.

14. Tan JM, Dawson TM. Parkin blushed by PINK1. Neuron 2006; 50: 527-529.

15. Exner N, Treske B, Paquet D, Holmstrom K, Schiesling C, Gispert S et al. Loss-of-function of human PINK1 results in mitochondrial pathology and can be rescued by parkin. $J$ Neurosci 2007; 27: 12413-12418.

16. Yang $Y$, Ouyang $Y$, Yang L, Beal MF, McQuibban A, Vogel $H$ et al. Pink1 regulates mitochondrial dynamics through interaction with the fission/fusion machinery. Proc Natl Acad Sci USA 2008; 105: 7070-7075.

17. Park J, Lee G, Chung J. The PINK1-Parkin pathway is involved in the regulation of mitochondrial remodeling process. Biochem Biophys Res Commun 2009; 378: 518-523.

18. Lutz AK, Exner N, Fett ME, Schlehe JS, Kloos K, Laemmermann K et al. Loss of parkin or PINK1 function increases DRP1-dependent mitochondrial fragmentation. J Biol Chem 2009; 284: 22938-22951.

19. Dagda RK, Cherra III SJ, Kulich SM, Tandon A, Park D, Chu CT. Loss of PINK1 function promotes mitophagy through effects on oxidative stress and mitochondrial fission. $J$ Biol Chem 2009; 284: 13843-13855.

20. Narendra D, Tanaka A, Suen DF, Youle RJ. Parkin is recruited selectively to impaired mitochondria and promotes their autophagy. J Cell Biol 2008; 183: 795-803.

21. Kim I, Rodriguez-Enriquez S, Lemasters JJ. Selective degradation of mitochondria by mitophagy. Arch Biochem Biophys 2007; 462: 245-253.

22. Komatsu M, Ueno T, Waguri S, Uchiyama Y, Kominami E, Tanaka K. Constitutive autophagy: vital role in clearance of unfavorable proteins in neurons. Cell Death Differ 2007; 14: 887-894.

23. Martinez-Vicente M, Cuervo AM. Autophagy and neurodegeneration: when the cleaning crew goes on strike. Lancet Neurol 2007; 6: 352-361.

24. Winslow AR, Rubinsztein DC. Autophagy in neurodegeneration and development. Biochim Biophys Acta 2008; 1782: 723-729. 
25. Gomes LC, Scorrano L. High levels of Fis1, a pro-fission mitochondrial protein, trigger autophagy. Biochim Biophys Acta 2008; 1777: 860-866.

26. Twig G, Hyde B, Shirihai OS. Mitochondrial fusion, fission and autophagy as a quality control axis: the bioenergetic view. Biochim Biophys Acta 2008; 1777: 1092-1097.

27. Weihofen A, Thomas KJ, Ostaszewski BL, Cookson MR, Selkoe DJ. Pink1 forms a multiprotein complex with Miro and Milton, linking Pink1 function to mitochondrial trafficking (dagger). Biochemistry 2009; 48: 2045-2052.

28. Silvestri L, Caputo V, Bellacchio E, Atorino L, Dallapiccola B, Valente EM et al. Mitochondrial import and enzymatic activity of PINK1 mutants associated to recessive Parkinsonism. Hum Mol Genet 2005; 14: 3477-3492.

29. Cao Y, Klionsky DJ. Physiological functions of Atg6/Beclin 1: a unique autophagy-related protein. Cell Res 2007; 17: 839-849.

30. Matsunaga K, Saitoh T, Tabata K, Omori H, Satoh T, Kurotori N et al. Two Beclin 1-binding proteins, Atg14L and Rubicon, reciprocally regulate autophagy at different stages. Nat Cell Biol 2009; 11: 385-396.

31. Gimenez-Xavier P, Francisco R, Santidrian AF, Gil J, Ambrosio S. Effects of dopamine on LC3-II activation as a marker of autophagy in a neuroblastoma cell model. Neurotoxicology 2009; 30: 658-665.

32. Mizushima N, Yoshimori T. How to interpret LC3 immunoblotting. Autophagy 2007; 3 542-545.

33. Klionsky DJ, Cuervo AM, Seglen PO. Methods for monitoring autophagy from yeast to human. Autophagy 2007; 3: 181-206.

34. Cipolat S, Martins de BO, Dal ZB, Scorrano L. OPA1 requires mitofusin 1 to promote mitochondrial fusion. Proc Natl Acad Sci USA 2004; 101: 15927-15932.
35. Pattingre S, Tassa A, Qu X, Garuti R, Liang XH, Mizushima N et al. Bcl-2 antiapoptotic proteins inhibit Beclin 1-dependent autophagy. Cell 2005; 122: 927-939.

36. Pickford F, Masliah E, Britschgi M, Lucin K, Narasimhan R, Jaeger PA et al. The autophagy-related protein beclin 1 shows reduced expression in early Alzheimer disease and regulates amyloid beta accumulation in mice. J Clin Invest 2008; 118: 2190-2199.

37. Shibata M, Lu T, Furuya T, Degterev A, Mizushima N, Yoshimori T et al. Regulation of intracellular accumulation of mutant Huntingtin by Beclin 1. J Biol Chem 2006; 281: 14474-14485.

38. Maiuri MC, Criollo A, Tasdemir E, Vicencio JM, Tajeddine N, Hickman JA et al. BH3-only proteins and $\mathrm{BH} 3$ mimetics induce autophagy by competitively disrupting the interaction between Beclin 1 and Bcl-2/Bcl-X(L). Autophagy 2007; 3: 374-376.

39. Zalckvar E, Berissi H, Mizrachy L, Idelchuk Y, Koren I, Eisenstein M et al. DAP-kinasemediated phosphorylation on the $\mathrm{BH} 3$ domain of beclin 1 promotes dissociation of beclin 1 from $\mathrm{Bcl}-\mathrm{X}(\mathrm{L})$ and induction of autophagy. EMBO Rep 2009; 10: 285-292.

40. Petiot A, Ogier-Denis E, Blommaart EF, Meijer AJ, Codogno P. Distinct classes of phosphatidylinositol $3^{\prime}$-kinases are involved in signaling pathways that control macroautophagy in HT-29 cells. J Biol Chem 2000; 275: 992-998.

41. Nishida Y, Arakawa S, Fujitani K, Yamaguchi H, Mizuta T, Kanaseki T et al. Discovery of Atg5/Atg7-independent alternative macroautophagy. Nature 2009; 461: 654-658.

42. Chu CT, Zhu J, Dagda R. Beclin 1-independent pathway of damage-induced mitophagy and autophagic stress: implications for neurodegeneration and cell death. Autophagy 2007; 3: 663-666.

43. Semple JI, Prime G, Wallis LJ, Sanderson CM, Markie D. Two-hybrid reporter vectors for gap repair cloning. Biotechniques 2005; 38: 927-934.

44. Hallworth R, Currall B, Nichols MG, Wu X, Zuo J. Studying inner ear protein-protein interactions using FRET and FLIM. Brain Res 2006; 1091: 122-131.

\section{Supplementary Information accompanies the paper on Cell Death and Differentiation website (http://www.nature.com/cdd)}

